\title{
TYPE IA SUPERNOVAE AND COSMOLOGY
}

\author{
Alexei V. Filippenko \\ Department of Astronomy, University of California, \\ Berkeley, CA 94720-3411, USA \\ alex@astro.berkeley.edu
}

\begin{abstract}
I discuss the use of Type Ia supernovae (SNe Ia) for cosmological distance determinations. Low-redshift $\mathrm{SNe}$ Ia $(z \lesssim 0.1)$ demonstrate that the Hubble expansion is linear with $H_{0}=72 \pm 8 \mathrm{~km} \mathrm{~s}^{-1} \mathrm{Mpc}^{-1}$, and that the properties of dust in other galaxies are generally similar to those of dust in the Milky Way. The light curves of high-redshift $\left(z=0.3^{-}\right.$ 1) SNe Ia are stretched in a manner consistent with the expansion of space; similarly, their spectra exhibit slower temporal evolution (by a factor of $1+z$ ) than those of nearby SNe Ia. The measured luminosity distances of SNe Ia as a function of redshift have shown that the expansion of the Universe is currently accelerating, probably due to the presence of repulsive dark energy such as Einstein's cosmological constant $(\Lambda)$. From about $200 \mathrm{SNe} \mathrm{Ia}$, we find that $H_{0} t_{0}=0.96 \pm 0.04$, and $\Omega_{\Lambda}-1.4 \Omega_{M}=0.35 \pm 0.14$. Combining our data with the results of large-scale structure surveys, we find a best fit for $\Omega_{M}$ and $\Omega_{\Lambda}$ of 0.28 and 0.72 , respectively — essentially identical to the recent WMAP results (and having comparable precision). The sum of the densities, $\sim 1.0$, agrees with extensive measurements of the cosmic microwave background radiation, including $W M A P$, and coincides with the value predicted by most inflationary models for the early Universe: the Universe is flat on large scales. A number of possible systematic effects (dust, supernova evolution) thus far do not seem to eliminate the need for $\Omega_{\Lambda}>0$. However, during the past few years some very peculiar low-redshift SNe Ia have been discovered, and we must be mindful of possible systematic effects if such objects are more abundant at high redshifts. Recently, analyses of SNe Ia at $z=1.0-1.7$ provide further support for current acceleration, and give tentative evidence for an early epoch of deceleration. The dynamical age of the Universe is estimated to be $13.1 \pm 1.5 \mathrm{Gyr}$, consistent with the ages of globular star clusters and with the WMAP result of $13.7 \pm 0.2$ Gyr. According to the most recent data sets, the SN Ia rate at $z>1$ is several times greater than that at low redshifts, presumably because of higher star formation rates long ago. Moreover, the typical delay time from progenitor star formation to SN Ia explosion appears to be substantial, $\sim 3$ Gyr. Current projects include the search for additional SNe Ia at $z>1$ to confirm
\end{abstract}


the early deceleration, and the measurement of a few hundred SNe Ia at $z=0.2-0.8$ to more accurately determine the equation-of-state parameter of the dark energy, $w=P /\left(\rho c^{2}\right)$, whose value is now constrained by $\mathrm{SNe}$ Ia to be in the range $-1.48 \lesssim w \lesssim-0.72$ at $95 \%$ confidence.

Keywords: cosmological parameters, dark energy, distance scale, supernovae

\section{Introduction}

Supernovae (SNe) come in two main observational varieties (see Filippenko 1997b for a review). Those whose optical spectra exhibit hydrogen are classified as Type II, while hydrogen-deficient $\mathrm{SNe}$ are designated Type I. SNe I are further subdivided according to the detailed appearance of the early-time spectrum: SNe Ia are characterized by strong absorption near $6150 \AA$ now attributed to Si II, SNe Ib lack this feature but instead show prominent He I lines, and SNe Ic have neither the Si II nor the He I lines (at least not strong ones). SNe Ia are believed to result from the thermonuclear disruption of carbon-oxygen white dwarfs, while SNe II come from core collapse in massive supergiant stars. The latter mechanism probably produces most $\mathrm{SNe} \mathrm{Ib} / \mathrm{Ic}$ as well, but the progenitor stars previously lost their outer layers of hydrogen or even helium, through either winds or mass transfer onto a companion star.

It has long been recognized that SNe Ia may be very useful distance indicators for a number of reasons; see Branch \& Tammann (1992), Branch (1998), and references therein. (1) They are exceedingly luminous, with peak $M_{B}$ averaging $-19.0 \mathrm{mag}$ if $H_{0}=72 \mathrm{~km} \mathrm{~s}^{-1} \mathrm{Mpc}^{-1}$. (2) "Normal" SNe Ia have small dispersion among their peak absolute magnitudes ( $\sigma \lesssim 0.3 \mathrm{mag}$ ). (3) Our understanding of the progenitors and explosion mechanism of SNe Ia is on a reasonably firm physical basis. The results of recent models give good fits to the observed spectra and light curves, providing confidence that we are not far off the mark. (4) Little cosmic evolution is expected in the peak luminosities of SNe Ia, and it can be modeled. (5) One can perform local tests of various possible complications and evolutionary effects by comparing nearby SNe Ia in different environments (elliptical galaxies, bulges and disks of spirals, galaxies having different metallicities, etc.).

Research on SNe Ia in the 1990s has demonstrated their enormous potential as distance indicators. Although there are subtle effects that must indeed be taken into account, it appears that SNe Ia provide among the most accurate values of $H_{0}, q_{0}$ (the deceleration parameter), $\Omega_{M}$ (the matter density), and $\Omega_{\Lambda}$ [the cosmological constant, $\left.\Lambda c^{2} /\left(3 H_{0}^{2}\right)\right]$.

For more than a decade there have been two major teams involved in the systematic investigation of high-redshift SNe Ia for cosmolog- 
ical purposes. The "Supernova Cosmology Project" (SCP) is led by Saul Perlmutter of the Lawrence Berkeley Laboratory, while the "HighZ supernova search Team" (HZT) is led by Brian Schmidt of the Mt. Stromlo and Siding Springs Observatories. I have been privileged to work with both of these teams (see Filippenko 2001 for a personal account), but my primary allegiance is now with the HZT. A few years ago, the HZT split into two overlapping subsets: the "Higher-Z Supernova Search Team" led by Adam Riess of the Space Telescope Science Institute, and the ESSENCE team ("Equation of State: SupErNovae trace Cosmic Expansion") led by Christopher Stubbs of Harvard University. Other groups have recently formed to conduct similar studies, such as the supernova team of the Canada-France-Hawaii Telescope Legacy Survey. An outgrowth of the SCP, the very large SNAP (SuperNova/Acceleration Probe) collaboration is planning a future space satellite that will be largely devoted to using SNe Ia for cosmology.

\section{Homogeneity and Heterogeneity}

Until the mid-1990s, the traditional way in which SNe Ia were used for cosmological distance determinations was to assume that they are perfect "standard candles" and to compare their observed peak brightness with that of SNe Ia in galaxies whose distances had been independently determined (e.g., with Cepheid variables). The rationale was that SNe Ia exhibit relatively little scatter in their peak blue luminosity $\left(\sigma_{B} \approx 0.4-\right.$ 0.5 mag; Branch \& Miller 1993), and even less if "peculiar" or highly reddened objects were eliminated from consideration by using a color cut. Moreover, the optical spectra of SNe Ia are usually rather homogeneous, if care is taken to compare objects at similar times relative to maximum brightness (Riess et al. 1997, and references therein). Over $80 \%$ of all SNe Ia discovered through the early 1990s were "normal" (Branch, Fisher, \& Nugent 1993).

From a Hubble diagram constructed with unreddened, moderately distant SNe Ia $(z \lesssim 0.1)$ for which peculiar motions are small and relative distances (given by ratios of redshifts) are accurate, Vaughan et al. (1995) find that

$$
\left\langle M_{B}(\max )\right\rangle=(-19.74 \pm 0.06)+5 \log \left(H_{0} / 50\right) \operatorname{mag}
$$

In a series of papers, Sandage et al. (1996) and Saha et al. (1997) combine similar relations with Hubble Space Telescope (HST) Cepheid distances to the host galaxies of seven SNe Ia to derive $H_{0}=57 \pm 4 \mathrm{~km}$ $\mathrm{s}^{-1} \mathrm{Mpc}^{-1}$. 
Over the past two decades it has become clear, however, that SNe Ia do not constitute a perfectly homogeneous subclass (e.g., Filippenko 1997a,b). In retrospect this should have been obvious: the Hubble diagram for SNe Ia exhibits scatter larger than the photometric errors, the dispersion actually rises when reddening corrections are applied (under the assumption that all SNe Ia have uniform, very blue intrinsic colors at maximum; van den Bergh \& Pazder 1992; Sandage \& Tammann 1993), and there are some significant outliers whose anomalous magnitudes cannot be explained by extinction alone.

Spectroscopic and photometric peculiarities have been noted with increasing frequency in well-observed SNe Ia. A striking case is SN 1991T; its pre-maximum spectrum did not exhibit Si II or Ca II absorption lines, yet two months past maximum brightness the spectrum was nearly indistinguishable from that of a classical SN Ia (Filippenko et al. 1992b; Ruiz-Lapuente et al. 1992; Phillips et al. 1992). The light curves of SN 1991T were slightly broader than the SN Ia template curves, and the object was probably somewhat more luminous than average at maximum. Another well-observed, peculiar SNe Ia is SN 1991bg (Filippenko et al. 1992a; Leibundgut et al. 1993; Turatto et al. 1996). At maximum brightness it was subluminous by $1.6 \mathrm{mag}$ in $V$ and $2.5 \mathrm{mag}$ in $B$, its colors were intrinsically red, and its spectrum was peculiar (with a deep absorption trough due to Ti II). Moreover, the decline from maximum was very steep, the $I$-band light curve did not exhibit a secondary maximum like normal SNe Ia, and the velocity of the ejecta was unusually low. The photometric heterogeneity among SNe Ia is well demonstrated by Suntzeff (1996) with objects having excellent BVRI light curves.

\section{Cosmological Uses: Low Redshifts}

Although SNe Ia can no longer be considered perfect "standard candles," they are still exceptionally useful for cosmological distance determinations. Excluding those of low luminosity (which are hard to find, especially at large distances), most of the nearby SNe Ia that had been discovered through the early 1990s were nearly standard (Branch et al. 1993; but see Li et al. 2001b for more recent evidence of a higher intrinsic peculiarity rate). Also, after many tenuous suggestions (e.g., Pskovskii 1977, 1984; Branch 1981), Phillips (1993) found convincing evidence for a correlation between light-curve shape and the luminosity at maximum brightness by quantifying the photometric differences among a set of nine well-observed SNe Ia, using a parameter $\left[\Delta m_{15}(B)\right]$ that measures the total drop (in $B$ magnitudes) from $B$-band maximum to $t=15$ days later. In all cases the host galaxies of his $\mathrm{SNe}$ Ia have accurate relative 
distances from surface brightness fluctuations or from the Tully-Fisher relation. The intrinsically bright $\mathrm{SNe}$ Ia clearly decline more slowly than dim ones, but the correlation is stronger in $B$ than in $V$ or $I$.

Using SNe Ia discovered during the Calán/Tololo survey $(z \lesssim 0.1)$, Hamuy et al. (1995, 1996b) refine the Phillips (1993) correlation between peak luminosity and $\Delta m_{15}(B)$. Apparently the slope is steep only at low luminosities; thus, objects such as SN 1991bg skew the slope of the best-fitting single straight line. Hamuy et al. reduce the scatter in the Hubble diagram of normal, unreddened SNe Ia to only 0.17 mag in $B$ and $0.14 \mathrm{mag}$ in $V$; see also Tripp (1997). Yet another parameterization is the "stretch" method of Perlmutter et al. (1997) and Goldhaber et al. (2001): the B-band light curves of SNe Ia appear nearly identical when expanded or contracted temporally by a factor $(1+s)$, where the value of $s$ varies among objects. In a similar but distinct effort, Riess, Press, \& Kirshner (1995) show that the luminosity of SNe Ia correlates with the detailed shape of the overall light curve.

By using light-curve shapes measured through several different filters, Riess, Press, \& Kirshner (1996a) extend their analysis and objectively eliminate the effects of interstellar extinction (as is also now done with the $\Delta m_{15}$ method; Phillips et al. 1999). A SN Ia that has an unusually red $B-V$ color at maximum brightness is assumed to be intrinsically subluminous if its light curves rise and decline quickly, or of normal luminosity but significantly reddened if its light curves rise and decline more slowly. With a set of 20 SNe Ia from the Calán/Tololo sample (Hamuy et al. 1996c) and the Harvard/Smithsonian Center for Astrophysics (CfA) follow-up program (Riess et al. 1999a), Riess et al. (1996a) show that the dispersion decreases from $0.52 \mathrm{mag}$ to $0.12 \mathrm{mag}$ after application of this "multi-color light-curve shape" (MLCS) method. The results from an expanded set of nearly $50 \mathrm{SNe}$ Ia indicate that the dispersion decreases from $0.44 \mathrm{mag}$ to $0.15 \mathrm{mag}$ (Figure 1). The resulting Hubble constant is $65 \pm 2$ (statistical) \pm 7 (systematic) $\mathrm{km} \mathrm{s}^{-1} \mathrm{Mpc}^{-1}$, with an additional systematic and zero-point uncertainty of $\pm 5 \mathrm{~km} \mathrm{~s}^{-1} \mathrm{Mpc}^{-1}$. (Re-calibrations of the Cepheid distance scale, and other recent refinements, lead to a best estimate of $H_{0}=72 \pm 8 \mathrm{~km} \mathrm{~s}^{-1} \mathrm{Mpc}^{-1}$, where the error bar includes both statistical and systematic uncertainties; Freedman et al. 2001.) Saha et al. (2001) still argue for a longer distance scale, with $H_{0} \approx 60 \mathrm{~km} \mathrm{~s}^{-1} \mathrm{Mpc}^{-1}$, using different choices in measuring the supernova distances (Parodi et al. 2000) and the Cepheid distances; see the discussions by Gibson et al. (2000) and Jha (2002).

Apart from the controversy regarding their true peak luminosity, lowredshift SNe Ia provide the best evidence that the Hubble flow is linear (Riess et al. 1996a), with deviations from linearity well-explained by 
random peculiar velocities and the local flow field (Riess et al. 1997; Jha 2002). Phillips et al. (1999) and Riess et al. (1996a) further argue that the dust affecting SNe Ia is not of circumstellar origin, and show quantitatively that the extinction curve in external galaxies typically does not differ from that in the Milky Way (cf. Branch \& Tammann 1992, but see Tripp 1998).

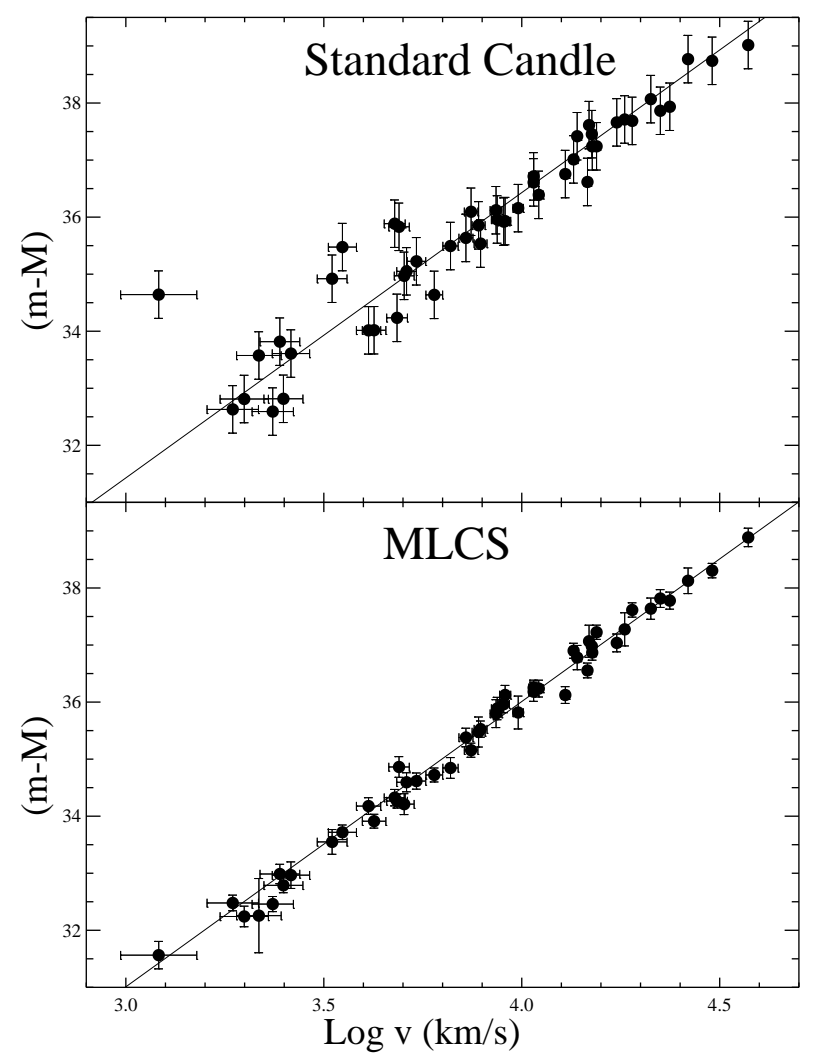

Figure 1: Hubble diagrams for SNe Ia (A. G. Riess 2001, private communication) with velocities $\left(\mathrm{km} \mathrm{s}^{-1}\right)$ in the $C O B E$ rest frame on the Cepheid distance scale. The ordinate shows distance modulus, $m-M$ (mag). Top: The objects are assumed to be standard candles and there is no correction for extinction; the result is $\sigma=0.42 \mathrm{mag}$ and $H_{0}=58 \pm 8$ $\mathrm{km} \mathrm{s}^{-1} \mathrm{Mpc}^{-1}$. Bottom: The same objects, after correction for reddening and intrinsic differences in luminosity. The result is $\sigma=0.15 \mathrm{mag}$ and $H_{0}=65 \pm 2$ (statistical) $\mathrm{km} \mathrm{s}^{-1} \mathrm{Mpc}^{-1}$, subject to changes in the zero-point of the Cepheid distance scale. (Indeed, the latest results with SNe Ia favor $H_{0}=72 \mathrm{~km} \mathrm{~s}^{-1} \mathrm{Mpc}^{-1}$.) 
The advantage of systematically correcting the luminosities of SNe Ia at high redshifts rather than trying to isolate "normal" ones seems clear in view of evidence that the luminosity of SNe Ia may be a function of stellar population. If the most luminous $\mathrm{SNe}$ Ia occur in young stellar populations (e.g., Hamuy et al. 1996a, 2000; Branch, Romanishin, \& Baron 1996; Ivanov, Hamuy, \& Pinto 2000), then we might expect the mean peak luminosity of high- $z$ SNe Ia to differ from that of a local sample. Alternatively, the use of Cepheids (Population I objects) to calibrate local SNe Ia can lead to a zero-point that is too luminous. On the other hand, as long as the physics of SNe Ia is essentially the same in young stellar populations locally and at high redshift, we should be able to adopt the luminosity correction methods (photometric and spectroscopic) found from detailed studies of low- $z$ SNe Ia.

In the past few years, many nearby SNe have been found by industrious amateur astronomers including R. Arbour, M. Armstrong, T. Boles, T. Puckett, M. Schwartz, and others. The "Nearby Supernova Factory" run by G. Aldering's team at the Lawrence Berkeley National Laboratory is also responsible for many discoveries, when it is conducting a search. However, about half of all reported nearby SNe in the last half-decade were discovered by my team's Lick Observatory Supernova Search (LOSS) conducted with the 0.76-m Katzman Automatic Imaging Telescope (KAIT; Li et al. 2000; Filippenko et al. 2001; Filippenko 2003; see http://astro.berkeley.edu/ bait/kait.html). During LOSS, CCD images are taken of $\gtrsim 1000$ galaxies per night and compared with KAIT "template images" obtained earlier; the templates are automatically subtracted from the new images and analyzed with computer software, and the SN candidates are flagged. The next day, undergraduate students at UC Berkeley examine all candidates, including weak ones, to eliminate star-like cosmic rays, asteroids, and other sources of false alarms. They also glance at some of the subtracted images to locate SNe that might be near bright, poorly subtracted stars or galactic nuclei. LOSS discovered $20 \mathrm{SNe}$ in 1998, 40 in 1999, 38 in 2000, 68 in 2001, 82 in 2002, and 95 in 2003, making it by far the world's leading search for nearby SNe.

Spectroscopic classifications of these and other low-redshift SNe are provided by a number of groups (see the IAU Circulars), including our own observations with the Lick 3-m and Keck telescopes, the CfA SN monitoring campaign, the Texas/McDonald Observatory group, the Asiago team, the European Research and Training Network, the Australian National University team, etc. The most important objects were photometrically monitored with KAIT through BVRI (and sometimes $U$ ) filters (e.g., Li et al. 2001a, 2003b; Modjaz et al. 2001; Leonard et al. 2002a,b; Foley et al. 2003), and unfiltered follow-up observations (e.g., 
Matheson et al. 2001) were made of almost all of them during the course of LOSS. Note that recently, KAIT has also been used to automatically monitor the optical afterglows of gamma-ray bursts (Li et al. 2003a,c; Matheson et al. 2003).

This growing sample of well-observed SNe Ia allows us to more precisely calibrate the distance determinations, as well as to look for correlations between the observed properties of the SNe and their environment (Hubble type of host galaxy, metallicity, stellar population, etc.). Jha (2002) present UBVRI follow-up observations from the CfA SN monitoring campaign of 44 nearby $\mathrm{SNe}$ Ia (including many discovered by KAIT), from which they derive "MLCS2k2," an updated light-curve fitting technique that includes $U$-band templates (critical for SN Ia observations at high redshift). Recently, progress has also been made in the near-infrared, with Krisciunas et al. (2001, 2003, 2004) presenting $J H K$ light curves of nearby SNe Ia. These have enabled Krisciunas, Phillips, \& Suntzeff (2004) to construct near-infrared Hubble diagrams of SNe Ia, with significantly less effect from dust extinction along the line of sight. Their results suggest that SNe Ia are much closer to standard candles in the near-infrared than in the optical, with little dependence of light-curve shape on near-infrared luminosity.

\section{Cosmological Uses: High Redshifts}

These same techniques can be applied to construct a Hubble diagram with high-redshift SNe Ia, from which the value of $q_{0}=\left(\Omega_{M} / 2\right)-\Omega_{\Lambda}$ can be determined. With enough objects spanning a range of redshifts, we can measure $\Omega_{M}$ and $\Omega_{\Lambda}$ independently (e.g., Goobar \& Perlmutter 1995). Contours of peak apparent $R$-band magnitude for SNe Ia at two

redshifts have different slopes in the $\Omega_{M}-\Omega_{\Lambda}$ plane, and the regions of intersection provide the answers we seek.

\subsection{The Search}

Based on the pioneering work of Norgaard-Nielsen et al. (1989), whose goal was to find $\mathrm{SNe}$ in moderate-redshift clusters of galaxies, the SCP (Perlmutter et al. 1995a, 1997) and the HZT (Schmidt et al. 1998) devised a strategy that almost guarantees the discovery of many faint, distant SNe Ia "on demand," during a predetermined set of nights. This "batch" approach to studying distant SNe allows follow-up spectroscopy and photometry to be scheduled in advance, resulting in a systematic study not possible with random discoveries. Most of the searched fields are equatorial, permitting follow-up from both hemispheres. The SCP 
was the first group to convincingly demonstrate the ability to find $\mathrm{SNe}$ in batches.

Our approach is simple in principle; see Schmidt et al. (1998) for details, and for a description of our first high-redshift SN Ia (SN 1995K). Pairs of first-epoch images during the nights around new moon are obtained with wide-field cameras on large telescopes (e.g., the Big Throughput Camera on the CTIO 4-m Blanco telescope, and more recently the CTIO Mosaic II, the CFHT $8 \mathrm{~K}$ and $12 \mathrm{~K}$ mosaics, and Suprime Cam on Subaru), followed by second-epoch images 3-4 weeks later. (Pairs of images permit removal of cosmic rays, asteroids, and Kuiper-belt objects.) These are compared immediately using well-tested software, and new SN candidates are identified in the second-epoch images (Figure 2 ). Spectra are obtained as soon as possible after discovery to verify that the objects are SNe Ia and to determine their redshifts. Each team has already found over 200 SNe in concentrated batches, as reported in numerous IAU Circulars (e.g., Perlmutter et al. 1995b, 11 SNe with $0.16 \lesssim z \lesssim 0.65$; Suntzeff et al. 1996, 17 SNe with $0.09 \lesssim z \lesssim 0.84$ ).

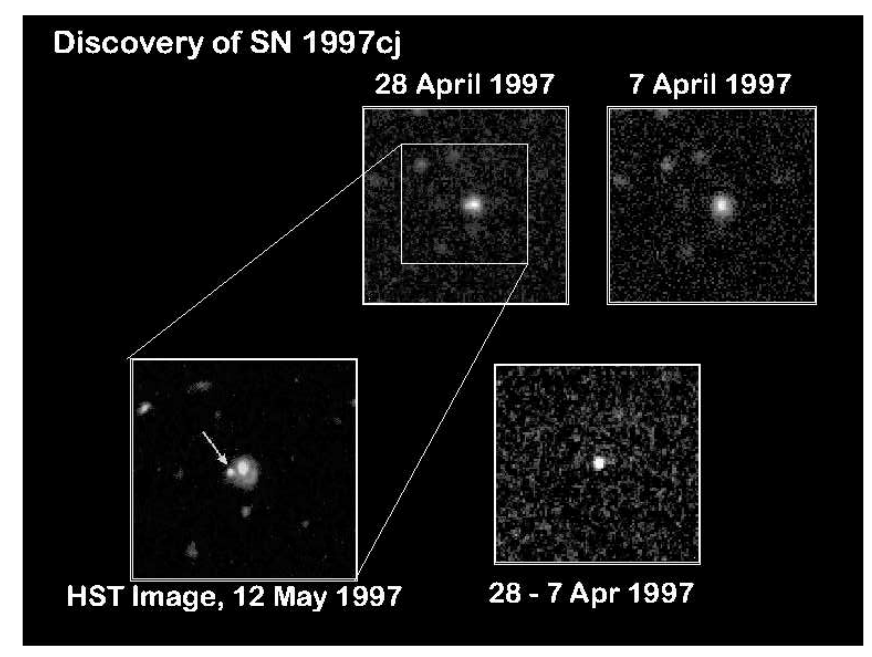

Figure 2: Discovery image of SN 1997cj (28 April 1997), along with the template image and an $H S T$ image obtained subsequently. The net (subtracted) image is also shown.

Intensive photometry of the SNe Ia commences within a few days after procurement of the second-epoch images; it is continued throughout the ensuing and subsequent dark runs. In a few cases HST images are obtained. As expected, most of the discoveries are on the rise or near maximum brightness. When possible, the SNe are observed in filters that 
closely match the redshifted $B$ and $V$ bands; this way, the K-corrections become only a second-order effect (Kim, Goobar, \& Perlmutter 1996; Nugent, Kim, \& Perlmutter 2002). Observations through two filters allow us to apply reddening and luminosity corrections (Riess et al. 1996a; Hamuy et al. 1996a,b; Phillips et al. 1999).

Although SNe in the magnitude range 22-22.5 can sometimes be spectroscopically confirmed with 4-m class telescopes, the signal-to-noise ratios are low, even after several hours of integration. Certainly Keck, Gemini, the VLT, Subaru, or Magellan are required for the fainter objects (22.5-24.5 mag). With the largest telescopes, not only can we rapidly confirm a substantial number of candidate SNe, but we can search for peculiarities in the spectra that might indicate evolution of SNe Ia with redshift. Moreover, high-quality spectra allow us to measure the age of a $\mathrm{SN}$ : we have developed a method for automatically comparing the spectrum of a SN Ia with a library of spectra from many different epochs in the development of SNe Ia (Riess et al. 1997). Our technique also has great practical utility at the telescope: we can determine the age of a SN "on the fly," within half an hour after obtaining its spectrum. This allows us to decide rapidly which SNe are best for subsequent photometric follow-up, and we immediately alert our collaborators elsewhere.

\subsection{Results}

First, we note that the light curves of high-redshift SNe Ia are broader than those of nearby SNe Ia; the initial indications (Leibundgut et al. 1996; Goldhaber et al. 1997), based on small numbers of SNe Ia, are amply confirmed with the larger samples (Goldhaber et al. 2001). Quantitatively, the amount by which the light curves are "stretched" is consistent with a factor of $1+z$, as expected if redshifts are produced by the expansion of space rather than by "tired light" and other non-expansion hypotheses for the redshifts of objects at cosmological distances. [For non-standard cosmological interpretations of the SN Ia data, see Narlikar \& Arp (1997) and Hoyle, Burbidge, \& Narlikar (2000).] We also demonstrate this spectroscopically at the $2 \sigma$ confidence level for a single object: the spectrum of SN 1996bj ( $z=0.57$ ) evolved more slowly than those of nearby SNe Ia, by a factor consistent with $1+z$ (Riess et al. 1997). Observations of SN 1997ex $(z=0.36)$ at three epochs conclusively verify the effects of time dilation: temporal changes in the spectra are slower than those of nearby SNe Ia by roughly the expected factor of 1.36. Although one might be able to argue that something other than universal expansion could be the cause of the apparent stretching of SN Ia light 
curves at high redshifts, it is much more difficult to attribute apparently slower evolution of spectral details to an unknown effect.

The formal value of $\Omega_{M}$ derived from SNe Ia has changed with time. The SCP published the initial result (Perlmutter et al. 1995a), based on a single object, SN 1992bi at $z=0.458: \Omega_{M}=0.2 \pm 0.6 \pm 1.1$ (assuming that $\Omega_{\Lambda}=0$ ). The SCP's analysis of their first seven objects (Perlmutter et al. 1997) suggested a much larger value of $\Omega_{M}=0.88 \pm 0.6$ (if $\Omega_{\Lambda}=0$ ) or $\Omega_{M}=0.94 \pm 0.3$ (if $\Omega_{\text {total }}=1$ ). Such a high-density universe seemed at odds with other, independent measurements of $\Omega_{M}$ at that time. However, with the subsequent inclusion of just one more object, SN 1997ap at $z=0.83$ (the highest then known for a SN Ia; Perlmutter et al. 1998), their estimates were revised back down to $\Omega_{M}=0.2 \pm 0.4$ if $\Omega_{\Lambda}=0$, and $\Omega_{M}=0.6 \pm 0.2$ if $\Omega_{\text {total }}=1$; the apparent brightness of SN 1997ap had been precisely measured with HST, so it substantially affected the best fits.

Meanwhile, the HZT published (Garnavich et al. 1998a) an analysis of four objects (three of them observed with HST), including SN 1997ck at $z=0.97$, at that time a redshift record, although they cannot be absolutely certain that the object was a SN Ia because the spectrum is too poor. From these data, the HZT derived that $\Omega_{M}=-0.1 \pm$ 0.5 (assuming $\Omega_{\Lambda}=0$ ) and $\Omega_{M}=0.35 \pm 0.3$ (assuming $\Omega_{\text {total }}=1$ ), inconsistent with the high $\Omega_{M}$ initially found by Perlmutter et al. (1997) but consistent with the revised estimate in Perlmutter et al. (1998). An independent analysis of 10 SNe Ia using the "snapshot" distance method (with which conclusions are drawn from sparsely observed SNe Ia) gave quantitatively similar conclusions (Riess et al. 1998a). However, none of these early data sets carried the statistical discriminating power to detect cosmic acceleration.

The SCP's next results were announced at the 1998 January AAS meeting in Washington, DC. A press conference was scheduled, with the stated purpose of presenting and discussing the then-current evidence for a low- $\Omega_{M}$ universe as published by Perlmutter et al. (1998; SCP) and Garnavich et al. (1998a; HZT). When showing the SCP's Hubble diagram for SNe Ia, however, Perlmutter also pointed out tentative evidence for acceleration! He said that the conclusion was uncertain, and that the data were equally consistent with no acceleration; the systematic errors had not yet been adequately assessed. Essentially the same conclusion was given by the SCP in February 1998 during their talks at a major conference on dark matter held near Los Angeles (Goldhaber \& Perlmutter 1998).

Although it chose not to reveal them at the same 1998 January AAS meeting, the HZT already had similar, tentative evidence for acceleration 
in their own SN Ia data set. The HZT continued to perform numerous checks of their data analysis and interpretation, including fairly thorough consideration of various possible systematic effects. Unable to find any significant problems, even with the possible systematic effects, the HZT reported detection of a nonzero value for $\Omega_{\Lambda}$ (based on 16 high- $z$ SNe Ia) at the Los Angeles dark matter conference in February 1998 (Filippenko \& Riess 1998), and soon thereafter submitted a formal paper that was published in September 1998 (Riess et al. 1998b).
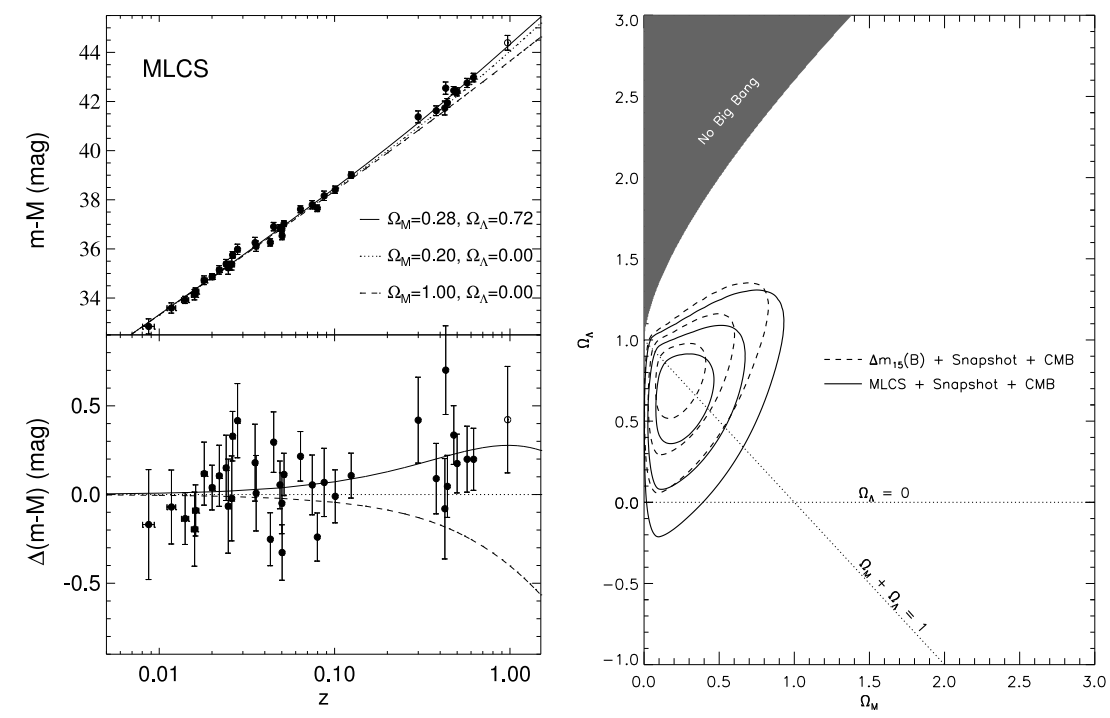

Figure 3 (left): The upper panel shows the Hubble diagram for the low$z$ and high- $z$ HZT SN Ia sample with MLCS distances; see Riess et al. (1998b). Overplotted are three world models: "low" and "high" $\Omega_{M}$ with $\Omega_{\Lambda}=0$, and the best fit for a flat universe $\left(\Omega_{M}=0.28, \Omega_{\Lambda}=0.72\right)$. The bottom panel shows the difference between data and models from the $\Omega_{M}=0.20, \Omega_{\Lambda}=0$ prediction. Only the 10 best-observed high- $z$ $\mathrm{SNe}$ Ia are shown. The average difference between the data and the $\Omega_{M}=0.20, \Omega_{\Lambda}=0$ prediction is $\sim 0.25 \mathrm{mag}$.

Figure 4 (right): The HZT's combined constraints from SNe Ia (left) and the position of the first acoustic peak of the cosmic microwave background (CMB) angular power spectrum, based on data available in mid-1998; see Garnavich et al. (1998b). The contours mark the 68.3\%, $95.4 \%$, and $99.7 \%$ enclosed probability regions. Solid curves correspond to results from the MLCS method; dotted ones are from the $\Delta m_{15}(B)$ method; all 16 SNe Ia in Riess et al. (1998b) were used. 
The HZT's original Hubble diagram for the 10 best-observed high- $z$ $\mathrm{SNe}$ Ia is given in Figure 3. With the MLCS method applied to the full set of $16 \mathrm{SNe}$ Ia, the HZT's formal results were $\Omega_{M}=0.24 \pm 0.10$ if $\Omega_{\text {total }}=1$, or $\Omega_{M}=-0.35 \pm 0.18$ (unphysical) if $\Omega_{\Lambda}=0$. If one demanded that $\Omega_{M}=0.2$, then the best value for $\Omega_{\Lambda}$ was $0.66 \pm 0.21$. These conclusions did not change significantly when only the 10 bestobserved SNe Ia were used (Figure $3 ; \Omega_{M}=0.28 \pm 0.10$ if $\Omega_{\text {total }}=1$ ).

Another important constraint on the cosmological parameters could be obtained from measurements of the angular scale of the first acoustic peak of the CMB (e.g., Zaldarriaga, Spergel, \& Seljak 1997; Eisenstein, $\mathrm{Hu}, \&$ Tegmark 1998); the SN Ia and CMB techniques provide nearly complementary information. A stunning result was already available by mid-1998 from existing measurements (e.g., Hancock et al. 1998; Lineweaver \& Barbosa 1998): the HZT's analysis of the SN Ia data in Riess et al. (1998b) demonstrated that $\Omega_{M}+\Omega_{\Lambda}=0.94 \pm 0.26$ (Figure 4 ), when the SN and CMB constraints were combined (Garnavich et al. 1998b; see also Lineweaver 1998, Efstathiou et al. 1999, and others).

Somewhat later (June 1999), the SCP published almost identical results (Perlmutter et al. 1999), implying an accelerating expansion of the Universe, based on an essentially independent set of 42 high- $z$ SNe (although a substantial number of these were later discarded because they are not clearly SNe Ia; Knop et al. 2003). The Perlmutter et al. (1999) data, together with those of the HZT, are shown in Figure 5, and the corresponding confidence contours in the $\Omega_{\Lambda}$ vs. $\Omega_{M}$ plane are given in Figure 6. This incredible agreement suggested that neither group had made a large, simple blunder; if the result was wrong, the reason must be subtle. Had there been only one team working in this area, it is likely that far fewer astronomers and physicists throughout the world would have taken the result seriously.

Moreover, already in 1998-1999 there was tentative evidence that the "dark energy" driving the accelerated expansion was indeed consistent with the cosmological constant, $\Lambda$. If $\Lambda$ dominates, then the equation-ofstate parameter of the dark energy should be $w=-1$, where the pressure $(P)$ and density $(\rho)$ are related according to $w=P /\left(\rho c^{2}\right)$. Garnavich et al. (1998b) and Perlmutter et al. (1999) were able to set an interesting limit, $w \lesssim-0.60$ at the $95 \%$ confidence level. However, more highquality data at $z \approx 0.5$ are needed to narrow the allowed range, in order to test other proposed candidates for dark energy such as various forms of "quintessence" (e.g., Caldwell, Davé, \& Steinhardt 1998).

Although the CMB results appeared reasonably persuasive in 19981999, one could argue that fluctuations on different scales had been measured with different instruments, and that subtle systematic ef- 
fects might lead to erroneous conclusions. These fears were dispelled only 1-2 years later, when the more accurate and precise results of the BOOMERANG collaboration were announced (de Bernardis et al. 2000, 2002). Shortly thereafter the MAXIMA collaboration distributed their very similar findings (Hanany et al. 2000; Balbi et al. 2000; Netterfield et al. 2002; see also the TOCO, DASI, and many other measurements). Figure 6 illustrates that the CMB measurements tightly constrain $\Omega_{\text {total }}$ to be close to unity; we appear to live in a flat universe, in agreement with most inflationary models for the early Universe! Combined with the SN Ia results, the evidence for nonzero $\Omega_{\Lambda}$ was fairly strong. Making the argument even more compelling was the fact that various studies of clusters of galaxies (see summary by Bahcall et al. 1999) showed that $\Omega_{M} \approx 0.3$, consistent with the results in Figures 4 and 6 . Thus, a "concordance cosmology" had emerged: $\Omega_{M} \approx 0.3, \Omega_{\Lambda} \approx 0.7-$ consistent with what had been suspected some years earlier by Ostriker \& Steinhardt (1995; see also Carroll, Press, \& Turner 1992).
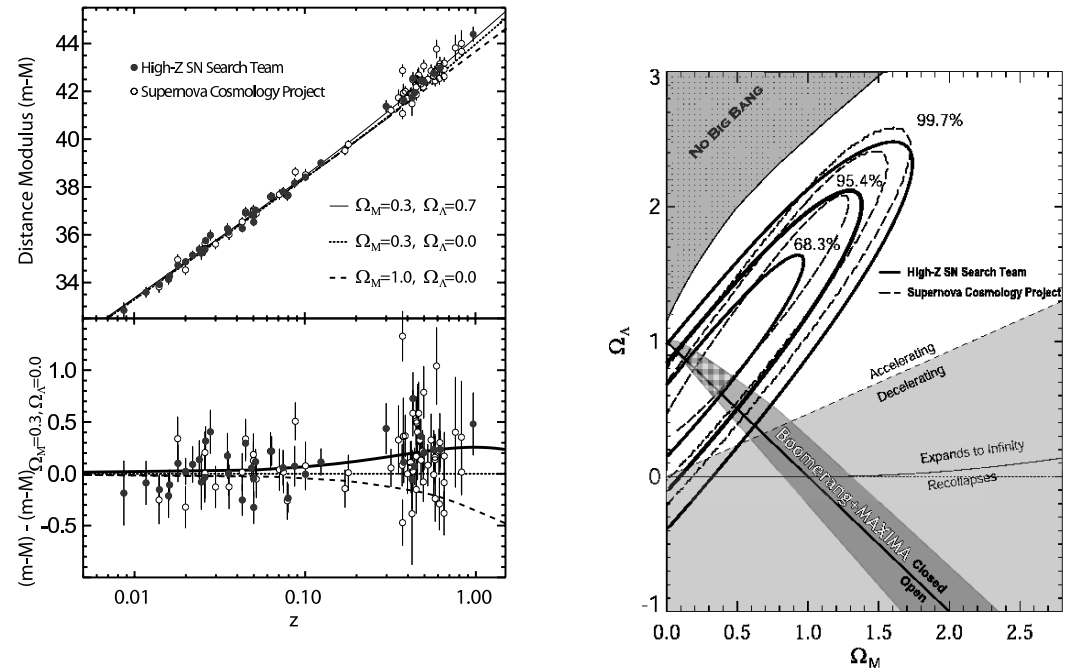

Figure 5 (left): As in Figure 3, but this time including both the HZT (Riess et al. 1998b) and SCP (Perlmutter et al. 1999) samples of lowredshift and high-redshift SNe Ia. Overplotted are three world models: $\Omega_{M}=0.3$ and 1.0 with $\Omega_{\Lambda}=0$, and a flat universe $\left(\Omega_{\text {total }}=1.0\right)$ with $\Omega_{\Lambda}=0.7$. The bottom panel shows the difference between data and models from the $\Omega_{M}=0.3, \Omega_{\Lambda}=0$ prediction.

Figure 6 (right): The combined constraints from SNe Ia (see Figure 5) and the position of the first acoustic peak of the CMB angular power spectrum, based on BOOMERANG and MAXIMA data. The contours mark the $68.3 \%, 95.4 \%$, and $99.7 \%$ enclosed probability regions determined from the SNe Ia. According to the $\mathrm{CMB}, \Omega_{\text {total }} \approx 1.0$. 
Yet another piece of evidence for a nonzero value of $\Lambda$ was provided by the Two-Degree Field Galaxy Redshift Survey (2dFGRS; Peacock et al. 2001; Percival et al. 2001; Efstathiou et al. 2002). Combined with the CMB maps, their results are inconsistent with a universe dominated by gravitating dark matter. Again, the implication is that about $70 \%$ of the mass-energy density of the Universe consists of some sort of dark energy whose gravitational effect is repulsive. In 2003, results from the first year of Wilkinson Microwave Anisotropy Probe (WMAP) observations appeared; together with the $2 \mathrm{dFGRS}$ constraints, they confirmed and refined the concordance cosmology $\left(\Omega_{M}=0.27, \Omega_{\Lambda}=0.73, \Omega_{\text {baryon }}=\right.$ $0.044, H_{0}=71 \pm 4 \mathrm{~km} \mathrm{~s}^{-1} \mathrm{Mpc}^{-1}$; Spergel et al. 2003, and references therein). Recent evidence for dark energy and an accelerating universe has also come from 2-3 $\sigma$ detections of the integrated Sachs-Wolfe effect (Afshordi, Loh, \& Strauss 2004; Boughn \& Crittenden 2004; Fosalba et al. 2003; Nolta et al. 2004; Scranton et al. 2004).

Meanwhile, the SN Ia measurements were becoming more numerous and of higher quality. For the HZT, the new (Fall 1999) sample of highredshift SNe Ia presented by Tonry et al. (2003), analyzed with methods distinct from (but similar to) those used previously, confirmed the result of Riess et al. (1998b) and Perlmutter et al. (1999) that the expansion of the Universe is accelerating. By combining all of the data sets available at that time, Tonry et al. (2003) were able to use about 200 SNe Ia, obtaining an incredibly firm detection of $\Omega_{\Lambda}>0$. They placed the following constraints on cosmological quantities: (1) If the equation-ofstate parameter of the dark energy is $w=-1$, then $H_{0} t_{0}=0.96 \pm 0.04$, and $\Omega_{\Lambda}-1.4 \Omega_{M}=0.35 \pm 0.14$. (2) Including the constraint of a flat universe, they find that $\Omega_{M}=0.28 \pm 0.05$, independent of any large-scale structure measurements. (3) Adopting a prior based on the 2dFGRS constraint on $\Omega_{M}$ (Percival et al. 2001) and assuming a flat universe, they derive that $-1.48<w<-0.72$ at $95 \%$ confidence. (4) Adopting the $2 \mathrm{dFGRS}$ results, they find $\Omega_{M}=0.28$ and $\Omega_{\Lambda}=0.72$, independent of any assumptions about $\Omega_{\text {total }}$. These constraints are similar in precision and in value to conclusions reported using Year 1 of WMAP (Spergel et al. 2003), also in combination with the 2dFGRS.

Even more recently, Barris et al. (2004) describe results from the 2001 HZT campaign. 23 SNe with $z=0.34-1.03$ were studied, 9 of which are unambiguously SNe Ia. The sample includes 15 objects at $z>0.7$, doubling the number of published $\mathrm{SNe}$ at these redshifts. Under the assumption that $\Omega_{\text {total }}=1$, they determine best-fit values of $\left(\Omega_{M}, \Omega_{\Lambda}\right)$ $=(0.33,0.67)$, using $22 \mathrm{SNe}$ from the $2001 \mathrm{HZT}$ survey together with 172 SNe from Tonry et al. (2003) satisfying $z>0.01$ and $A_{V} \leq 0.5$ mag. 
The SCP's high-quality HST data set on 11 SNe Ia in the redshift range $0.36-0.86$, recently published by Knop et al. (2003), independently confirms the apparent acceleration of the Universe. They were able to measure accurate colors of the SNe, providing better host-galaxy extinction estimates than had been possible in the past for individual objects. Thus, there was no need to make any assumptions or priors on the parent distribution of extinction values, $E(B-V)$, unlike the case in the initial analyses (Riess et al. 1998; Perlmutter et al. 1999; but see Sullivan et al. 2003, discussed below). Their extinction measurements do not show evidence for anomalously blue SNe Ia at high redshifts (or for a preponderance of negative $E(B-V)$ values for highredshift SNe Ia), contrary to some earlier suspicions (Falco et al. 1999; Leibundgut 2001). Knop et al. (2003) find that dark energy is required with a probability exceeding $99 \%$, consistent with previous studies that had made assumptions about the distribution of extinctions or that had used low-extinction subsets of SNe Ia. In a flat universe with a constant dark energy equation-of-state parameter $w=-1$, they find that $\Omega_{\Lambda}=0.75(+0.06,-0.07) \pm 0.04$, where the first quoted uncertainties are statistical and the second are identified systematics. Moreover, in a flat universe with $w$ constant in time, their SNe Ia data show that $w=-1.05(+0.15,-0.20) \pm 0.09$, consistent with $\Lambda$ (rather than with some quintessence models; see below).

The dynamical age of the Universe can be calculated from the cosmological parameters. In an empty Universe with no cosmological constant, the dynamical age is simply the inverse of the Hubble constant, $t_{0}=H_{0}^{-1}$; there is no deceleration. In the late-1990s, SNe Ia gave $H_{0}=65 \pm 7 \mathrm{~km} \mathrm{~s}^{-1} \mathrm{Mpc}^{-1}$, and a Hubble time of $15.1 \pm 1.6$ Gyr. For a more complex cosmology, integrating the velocity of the expansion from the current epoch $(z=0)$ to the beginning $(z=\infty)$ yields an expression for the dynamical age. As shown in detail by Riess et al. (1998b), by mid-1998 the HZT had obtained a value of $14.2_{-0.8}^{+1.0}$ Gyr (with $H_{0}=65$ ) using the likely range for $\left(\Omega_{M}, \Omega_{\Lambda}\right)$ that they measured. (The precision was so high because their experiment was sensitive to roughly the difference between $\Omega_{M}$ and $\Omega_{\Lambda}$, and the dynamical age also varies in approximately this way.) Including the systematic uncertainty of the Cepheid distance scale, which may be up to $10 \%$, a reasonable estimate of the dynamical age was 14.2 \pm 1.7 Gyr (Riess et al. 1998b). Again, the SCP's result was very similar (Perlmutter et al. 1999), since it was based on nearly the same derived values for the cosmological parameters. The most recent results, reported by Tonry et al. (2003; see also Knop et al. 2003) and adopting $H_{0}=72 \pm 8 \mathrm{~km} \mathrm{~s}^{-1} \mathrm{Mpc}^{-1}$, give a dynamical age of 
$13.1 \pm 1.5$ Gyr for the Universe - again, in agreement with the WMAP result of $13.7 \pm 0.2$ Gyr.

This expansion age is also consistent with ages determined from various other techniques such as the cooling of white dwarfs (Galactic disk $>9.5$ Gyr; Oswalt et al. 1996), radioactive dating of stars via the thorium and europium abundances (15.2 \pm 3.7 Gyr; Cowan et al. 1997), and studies of globular clusters (10-15 Gyr, depending on whether Hipparcos parallaxes of Cepheids are adopted; Gratton et al. 1997; Chaboyer et al. 1998). The ages of the oldest stars no longer seem to exceed the expansion age of the Universe; the long-standing "age crisis" has evidently been resolved.

\section{Discussion}

Although the convergence of different methods on the same answer is reassuring, and suggests that the concordance cosmology is correct, it is important to vigorously test each method to make sure it is not leading us astray. Moreover, only through such detailed studies will the accuracy and precision of the methods improve, allowing us to eventually set better constraints on the equation-of-state parameter, $w$. Here I discuss the systematic effects that could adversely affect the SN Ia results.

High-redshift SNe Ia are observed to be dimmer than expected in an empty Universe (i.e., $\Omega_{M}=0$ ) with no cosmological constant. At $z \approx 0.5$, where the SN Ia observations have their greatest leverage on $\Lambda$, the difference between an $\Omega_{M}=0.3\left(\Omega_{\Lambda}=0\right)$ universe and a flat universe with $\Omega_{\Lambda}=0.7$ is only about $0.25 \mathrm{mag}$. Thus, we need to find out if chemical abundances, stellar populations, selection bias, gravitational lensing, or grey dust can have an effect this large. Although both the HZT and SCP had considered many of these potential systematic effects in their original discovery papers (Riess et al. 1998b; Perlmutter et al. 1999), and had shown with reasonable confidence that obvious ones were not greatly affecting their conclusions, it was of course possible that they were wrong, and that the data were being misinterpreted.

\subsection{Gravitational Lensing}

The magnification and demagnification of light by large-scale structure can alter the observed magnitudes of high-redshift SNe (e.g., Kantowski, Vaughan, \& Branch 1995; Kantowski 1998). The effect of weak gravitational lensing on our analysis has been quantified by Wambsganss, Cen, \& Ostriker (1998) and summarized by Schmidt et al. (1998). SN Ia light will, on average, be demagnified by $0.5 \%$ at $z=0.5$ and by $1 \%$ at $z=1$ in a Universe with a non-negligible cosmological constant. Al- 
though the sign of the effect is the same as the influence of a cosmological constant, the size of the effect is small enough to be ignored.

Holz (1998), Holz \& Wald (1998), and Kantowski (1998) have calculated the weak lensing effects on supernova light from ordinary matter which is not smoothly distributed in galaxies but rather clumped into stars (i.e., dark matter contained in massive compact halo objects). With this scenario, microlensing becomes a more important effect, further decreasing the typical observed supernova luminosities at $z=0.5$ by $0.02 \mathrm{mag}$ for $\Omega_{M}=0.2$. Even if most ordinary matter were contained in compact objects, this effect would not be large enough to reconcile the SN Ia distances with the influence of ordinary matter alone in a decelerating universe. Barris et al. (2004), for example, show that use of the "empty-beam model" for gravitational lensing does not eliminate the need for $\Omega_{\Lambda}>0$. However, gravitational lensing will certainly need to be taken into account when making a precise measurement of $w$.

With a very large sample (200-1000) of high-redshift SNe Ia, and accurate photometry, it should be possible to quantify the effects of clumped matter. Light from some SNe Ia should be strongly amplified by the presence of intervening matter, while the vast majority will be deamplified (e.g., Barber et al. 2000). The distribution of amplification factors can be used to determine the type of dark matter most prevalent in the Universe (compact objects, or smoothly distributed).

\subsection{Evolution}

Perhaps the most obvious possible systematic effect is evolution of SNe Ia over cosmic time, due to changes in metallicity, progenitor mass, or some other factor. If the peak luminosity of $\mathrm{SNe}$ Ia were lower at high redshift, then the case for $\Omega_{\Lambda}>0$ would weaken. Conversely, if the distant explosions are more powerful, then the case for acceleration strengthens. Theorists are not yet sure what the sign of the effect will be, if it is present at all; different assumptions lead to different conclusions (Höflich, Wheeler, \& Thielemann 1998; Umeda et al. 1999; Nomoto et al. 2000; Yungelson \& Livio 2000).

Of course, it is extremely difficult, if not effectively impossible, to obtain an accurate, independent measure of the peak luminosity of high$z \mathrm{SNe}$ Ia, and hence to directly test for luminosity evolution. However, we can more easily determine whether other observable properties of low$z$ and high- $z$ SNe Ia differ. If they are all the same, it is more probable that the peak luminosity is constant as well - but if they differ, then the peak luminosity might also be affected (e.g., Höflich et al. 1998). Drell, Loredo, \& Wasserman (2000), for example, argue that there are 
reasons to suspect evolution, because the average properties of existing samples of high- $z$ and low- $z$ SNe Ia seem to differ (e.g., the high- $z$ SNe Ia are more uniform).

The local sample of SNe Ia displays a weak correlation between lightcurve shape and host-galaxy type, in the sense that the most luminous SNe Ia with the broadest light curves only occur in late-type galaxies. Both early-type and late-type galaxies provide hosts for dimmer SNe Ia with narrower light curves (Hamuy et al. 1996a). The mean luminosity difference for SNe Ia in late-type and early-type galaxies is $\sim 0.3 \mathrm{mag}$. In addition, the $\mathrm{SN}$ Ia rate per unit luminosity is almost twice as high in late-type galaxies as in early-type galaxies at the present epoch (Cappellaro et al. 1997). These results may indicate an evolution of SNe Ia with progenitor age. Possibly relevant physical parameters are the mass, metallicity, and C/O ratio of the progenitor (Höflich et al. 1998).

We expect that the relation between light-curve shape and peak luminosity that applies to the range of stellar populations and progenitor ages encountered in the late-type and early-type hosts in our nearby sample should also be applicable to the range encountered in our distant sample. In fact, the age range for SN Ia progenitors in the nearby sample is likely larger than the change in mean progenitor age over the 4-6 Gyr lookback time to the high- $z$ sample. Thus, to first order at least, our local sample should correct the distances for progenitor or age effects.

We can place empirical constraints on the effect that a change in the progenitor age would have on our SN Ia distances by comparing subsamples of low-redshift SNe Ia believed to arise from old and young progenitors. In the nearby sample, the mean difference between the distances for the early-type hosts ( $8 \mathrm{SNe}$ Ia) and late-type hosts (19 $\mathrm{SNe}$ Ia), at a given redshift, is $0.04 \pm 0.07 \mathrm{mag}$ from the MLCS method. This difference is consistent with zero. Even if the SN Ia progenitors evolved from one population at low redshift to the other at high redshift, we still would not explain the surplus in mean distance of 0.25 mag over the $\Omega_{\Lambda}=0$ prediction.

Moreover, in a major study of high-redshift SNe Ia as a function of galaxy morphology, the SCP found no clear differences (except for the amount of scatter; see $\S 5.2$ ) between the cosmological results obtained with SNe Ia in late-type and early-type galaxies (Sullivan et al. 2003). Similarly, a study of the host galaxies of high-redshift SNe Ia $(0.42<z<$ 1.06) done by the HZT (Williams et al. 2003) found no clear evidence for correlations between host-galaxy properties and the residuals of distance measurements from cosmological fits. Some of the correlations between SN Ia properties and host-galaxy type seen at low redshift also appear 
to be present at high redshift, again supporting the current practice of extrapolating properties of the nearby SN Ia population to high redshifts.

It is also reassuring that initial comparisons suggest that high- $z \mathrm{SN}$ Ia spectra appear similar to those observed at low redshift. For example, the spectral characteristics of SN 1998ai $(z=0.49)$ appear to be essentially indistinguishable from those of normal low- $z$ SNe Ia; see Figure 7. In fact, the most obviously discrepant spectrum in this figure is the second one, that of SN 1994B $(z=0.09)$; it is intentionally included as a "decoy" that illustrates the degree to which even the spectra of nearby, relatively normal SNe Ia can vary. Nevertheless, it is important to note that a dispersion in luminosity (perhaps $0.2 \mathrm{mag}$ ) exists even among the other, more normal SNe Ia shown in Figure 7; thus, our spectra of SN 1998ai and other high- $z$ SNe Ia are not yet sufficiently good for independent, precise determinations of peak luminosity from spectral features (Nugent et al. 1995). Many of them, however, are sufficient for ruling out other SN types (Figure 8), or for identifying gross peculiarities such as those shown by SNe 1991T and 1991bg; see Coil et al. (2000).

We can help verify that the SNe at $z \approx 0.5$ being used for cosmology do not belong to a subluminous population of SNe Ia by examining rest-frame $I$-band light curves. Normal, nearby SNe Ia show a pronounced second maximum in the $I$ band about a month after the first maximum and typically about 0.5 mag fainter (e.g., Ford et al. 1993; Suntzeff 1996). Subluminous SNe Ia, in contrast, do not show this second maximum, but rather follow a linear decline or show a muted second maximum (Filippenko et al. 1992a). As discussed by Riess et al. (2000), tentative evidence for the second maximum is seen from the HZT's existing $J$-band (rest-frame $I$-band) data on SN 1999Q $(z=0.46)$; see Figure 10. Additional tests with spectra and near-infrared light curves are currently being conducted.

Another way of using light curves to test for possible evolution of $\mathrm{SNe}$ Ia is to see whether the rise time (from explosion to maximum brightness) is the same for high-redshift and low-redshift SNe Ia; a difference might indicate that the peak luminosities are also different (Höflich et al. 1998). Riess et al. (1999c) measured the risetime of nearby SNe Ia, using data from KAIT, the Beijing Astronomical Observatory (BAO) SN search, and a few amateur astronomers. Though the exact value of the risetime is a function of peak luminosity, for typical low-redshift SNe Ia it is $20.0 \pm 0.2$ days. Riess et al. (1999b) pointed out that this differs by $5.8 \sigma$ from the preliminary risetime of $17.5 \pm 0.4$ days reported in conferences by the SCP (Goldhaber et al. 1998a,b; Groom 1998). However, more thorough analyses of the SCP data (Aldering, Knop, \& Nugent 2000; Goldhaber et al. 2001) show that the high-redshift uncertainty of 
\pm 0.4 days that the $\mathrm{SCP}$ originally reported was much too small because it did not account for systematic effects. The revised discrepancy with the low-redshift risetime is about $2 \sigma$ or less. Thus, the apparent difference in risetimes might be insignificant. Even if the difference is real, however, its relevance to the peak luminosity is unclear; the light curves may differ only in the first few days after the explosion, and this could be caused by small variations in conditions near the outer part of the exploding white dwarf that are inconsequential at the peak.
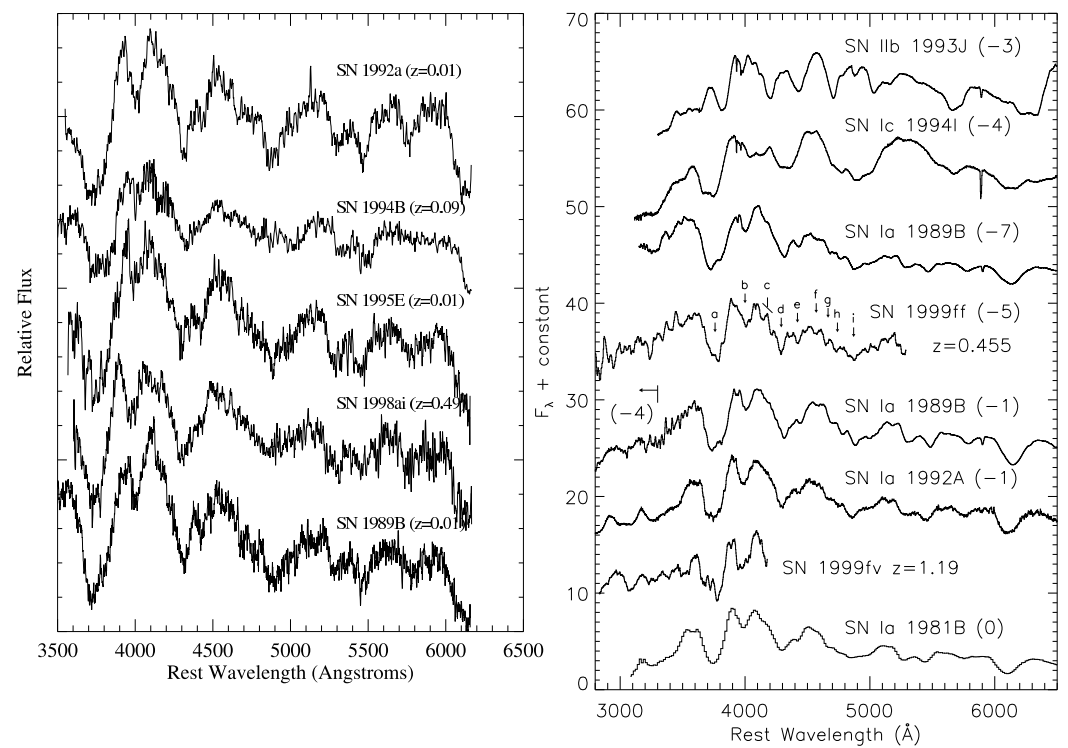

Figure 7 (left): Spectral comparison (in $f_{\lambda}$ ) of SN 1998ai $(z=0.49$; Keck spectrum) with low-redshift $(z<0.1)$ SNe Ia at a similar age $(\sim 5$ days before maximum brightness), from Riess et al. (1998b). The spectra of the low-redshift SNe Ia were resampled and convolved with Gaussian noise to match the quality of the spectrum of SN 1998ai. Overall, the agreement in the spectra is excellent, tentatively suggesting that distant SNe Ia are physically similar to nearby SNe Ia. SN 1994B $(z=0.09)$ differs the most from the others, and was included as a "decoy."

Figure 8 (right): Heavily smoothed spectra of two high- $z$ SNe (SN $1999 \mathrm{ff}$ at $z=0.455$ and SN $1999 \mathrm{fv}$ at $z=1.19$; quite noisy below $\sim 3500 \AA$ ) are presented along with several low- $z$ SN Ia spectra (SNe 1989B, 1992A, and 1981B), a SN Ib spectrum (SN 1993J), and a SN Ic spectrum (SN 1994I); see Filippenko (1997b) for a discussion of spectra of various types of SNe. The date of the spectra relative to $B$-band maximum is shown in parentheses after each object's name. Specific features seen in SN 1999ff and labeled with a letter are discussed by Coil et al. (2000). This comparison shows that the two high- $z$ SNe are most likely SNe Ia. 
Although there are no clear signs that cosmic evolution of SNe Ia seriously compromises our results, it is wise to remain vigilant for possible problems. At low redshifts, for example, we already know that some SNe Ia don't conform with the correlation between light curve shape and luminosity. SN 2000cx in the S0 galaxy NGC 524, for example, has light curves that cannot be fit well by any of the fitting techniques currently available ( $\mathrm{Li}$ et al. 2001a; Filippenko 2003); see Figure 9. Its late-time color is remarkably blue, inconsistent with the homogeneity described by Phillips et al. (1999). The spectral evolution of SN 2000cx is peculiar as well (Li et al. 2001a; Branch et al. 2004a): the photosphere appears to have remained hot for a long time, and both iron-peak and intermediate-mass elements move at very high velocities.
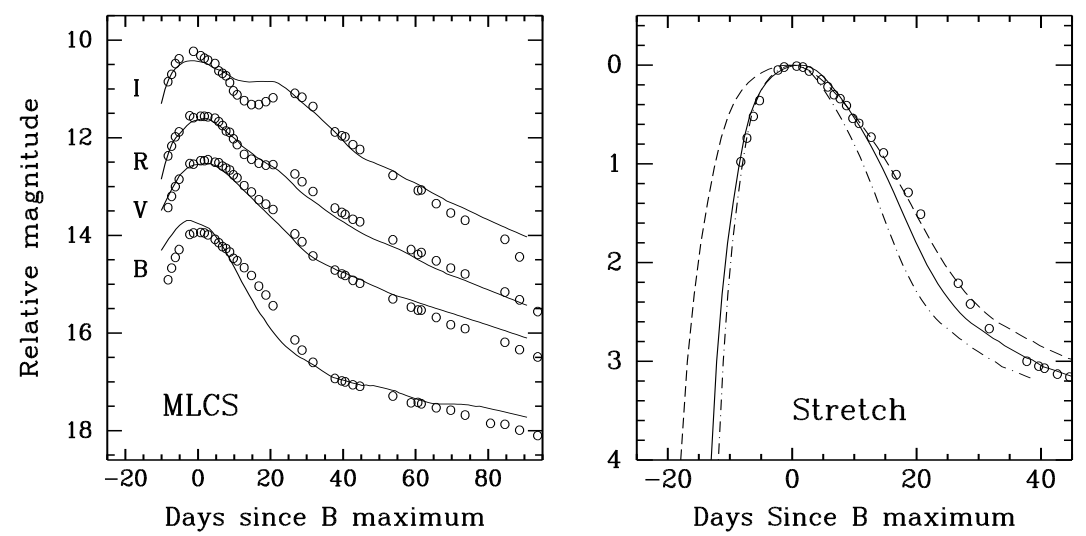

Figure 9: The MLCS fit (Riess et al. 1998b; left panel) and the stretch method fit (Perlmutter et al. 1999; right panel) for SN 2000cx. The MLCS fit is the worst we had ever seen through year 2000. For the stretch method fit, the solid line is the fit to all the data points from $t=-8$ to 32 days, the dash-dotted line uses only the premaximum datapoints, and the dashed line only the postmaximum datapoints. The three fits give very different stretch factors. From Li et al. (2001a).

An even more peculiar object is SN 2002cx (Li et al. 2003; Filippenko 2003; Branch et al. 2004b). It is spectroscopically bizarre, with extremely low expansion velocities and almost no evidence for intermediate-mass elements. The nebular phase was reached incredibly soon after maximum brightness, despite the low velocity of the ejecta, suggesting that the ejected mass is small. SN 2002cx was subluminous by $\sim 2$ mag at all optical wavelengths relative to normal SNe Ia, despite the early-time spectroscopic resemblance to the somewhat overluminous SN 1991T. The $R$-band and $I$-band light curves of SN 2002cx are com- 
pletely unlike those of normal SNe Ia. No existing theoretical model successfully explains all observed aspects of SN 2002cx, though 3D deflagration models may be best. If there are more strange beasts like $\mathrm{SNe}$ $2000 \mathrm{cx}$ and 2002cx at high redshifts than at low redshifts, systematic errors may creep into the analysis of high- $z$ SNe Ia.

\subsection{Extinction}

Our SN Ia distances have the important advantage of including corrections for interstellar extinction occurring in the host galaxy and the Milky Way. Extinction corrections based on the relation between SN Ia colors and luminosity improve distance precision for a sample of nearby SNe Ia that includes objects with substantial extinction (Riess et al. 1996a; Phillips et al. 1999); the scatter in the Hubble diagram is much reduced. Moreover, the consistency of the measured Hubble flow from SNe Ia with late-type and early-type hosts (see §5.1) shows that the extinction corrections applied to dusty SNe Ia at low redshift do not alter the expansion rate from its value measured from SNe Ia in low-dust environments.

In practice, the high-redshift SNe Ia generally appear to suffer very little extinction; their $B-V$ colors at maximum brightness are normal, suggesting little color excess due to reddening. The most detailed available study is that of the SCP (Sullivan et al. 2003): they found that the scatter in the Hubble diagram is minimal for SNe Ia in early-type host galaxies, but increases for SNe Ia in late-type galaxies. Moreover, on average the SNe in late-type galaxies are slightly fainter (by $0.14 \pm 0.09$ mag) than those in early-type galaxies. Finally, at peak brightness the colors of SNe Ia in late-type galaxies are marginally redder than those in early-type galaxies. Sullivan et al. (2003) conclude that extinction by dust in the host galaxies of SNe Ia is one of the major sources of scatter in the high-redshift Hubble diagram. By restricting their sample to $\mathrm{SNe}$ Ia in early-type host galaxies (presumably with minimal extinction), they obtain a very tight Hubble diagram that suggests a nonzero value for $\Omega_{\Lambda}$ at the $5 \sigma$ confidence level, under the assumption that $\Omega_{\text {total }}=1$. In the absence of this assumption, SNe Ia in early-type hosts still imply that $\Omega_{\Lambda}>0$ at nearly the $98 \%$ confidence level. The results for $\Omega_{\Lambda}$ with SNe Ia in late-type galaxies are quantitatively similar, but statistically less secure because of the larger scatter.

Riess, Press, \& Kirshner (1996b; see also Phillips et al. 1999) found indications that the Galactic ratios between selective absorption and color excess are similar for host galaxies in the nearby $(z \leq 0.1)$ Hubble flow. Yet, what if these ratios changed with lookback time (e.g., Aguirre 
1999a)? Could an evolution in dust-grain size descending from ancestral interstellar "pebbles" at higher redshifts cause us to underestimate the extinction? Large grains would not imprint the reddening signature of typical interstellar extinction upon which our corrections necessarily rely.
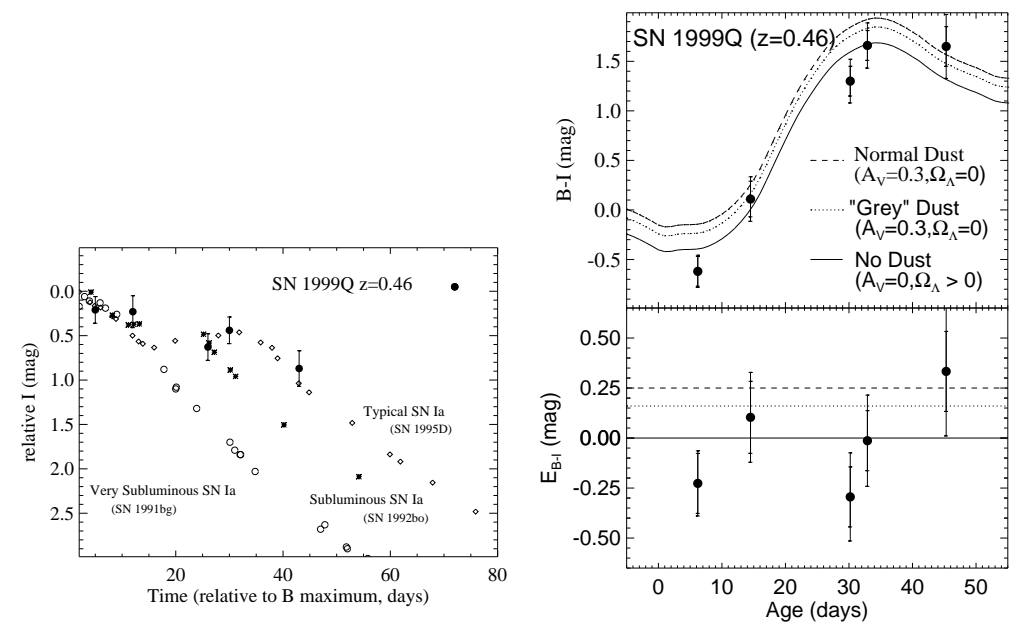

Figure 10 (left): Rest-frame $I$-band (observed $J$-band) light curve of SN 1999Q ( $z=0.46,5$ solid points; Riess et al. 2000), and the $I$-band light curves of several nearby SNe Ia. Subluminous SNe Ia exhibit a less prominent second maximum than do normal SNe Ia.

Figure 11 (right): Color $(B-I)$ and color excess $\left(E_{B-I}\right)$ for SN 1999Q and different dust models (Riess et al. 2000). The data are most consistent with no dust and $\Omega_{\Lambda}>0$.

However, viewing our SNe through such gray interstellar grains would also induce a dispersion in the derived distances. Using the results of Hatano, Branch, \& Deaton (1998), Riess et al. (1998b) estimate that the expected dispersion would be 0.40 mag if the mean gray extinction were $0.25 \mathrm{mag}$ (the value required to explain the measured MLCS distances without a cosmological constant). This is significantly larger than the $0.21 \mathrm{mag}$ dispersion observed in the high-redshift MLCS distances. Furthermore, most of the observed scatter is already consistent with the estimated statistical errors, leaving little to be caused by gray extinction. Nevertheless, if we assumed that all of the observed scatter were due to gray extinction, the mean shift in the SN Ia distances would be only 0.05 mag. With the existing observations, it is difficult to rule out this modest amount of gray interstellar extinction.

Gray intergalactic extinction could dim the SNe without either telltale reddening or dispersion, if all lines of sight to a given redshift had a similar column density of absorbing material. The component of the 
intergalactic medium with such uniform coverage corresponds to the gas clouds producing Lyman- $\alpha$ forest absorption at low redshifts. These clouds have individual H I column densities less than about $10^{15} \mathrm{~cm}^{-2}$ (Bahcall et al. 1996). However, they display low metallicities, typically less than $10 \%$ of solar. Gray extinction would require larger dust grains which would need a larger mass in heavy elements than typical interstellar grain size distributions to achieve a given extinction. It is possible that large dust grains are blown out of galaxies by radiation pressure, and are therefore not associated with Lyman- $\alpha$ clouds (Aguirre 1999b).

But even the dust postulated by Aguirre (1999a,b) and Aguirre \& Haiman (1999) is not completely gray, having a size of about $0.1 \mu \mathrm{m}$. We can test for such nearly gray dust by observing high-redshift SNe Ia over a wide wavelength range to measure the color excess it would introduce. If $A_{V}=0.25 \mathrm{mag}$, then $E(U-I)$ and $E(B-I)$ should be $0.12-0.16 \mathrm{mag}$ (Aguirre 1999a,b). If, on the other hand, the $0.25 \mathrm{mag}$ faintness is due to $\Lambda$, then no such reddening should be seen. This effect is measurable using proven techniques; so far, with just one SN Ia (SN 1999Q; Figure 11), our results favor the no-dust hypothesis to better than $2 \sigma$ (Riess et al. 2000). More work along these lines is in progress.

\subsection{Early Deceleration of the Universe}

Suppose, however, that for some reason the dust is very gray, or our color measurements are not sufficiently precise to rule out Aguirre's (or other) dust. Or, perhaps some other astrophysical systematic effect is fooling us, such as possible evolution of the white dwarf progenitors (e.g., Höflich et al. 1998; Umeda et al. 1999), or gravitational lensing (Wambsganss et al. 1998). The most decisive test to distinguish between $\Lambda$ and cumulative systematic effects is to examine the deviation of the observed peak magnitude of SNe Ia from the magnitude expected in the low- $\Omega_{M}$, zero- $\Lambda$ model. If $\Lambda$ is positive, the deviation should actually begin to decrease at $z \approx 1$; we will be looking so far back in time that the $\Lambda$ effect becomes small compared with $\Omega_{M}$, and the Universe is decelerating at that epoch. If, on the other hand, a systematic bias such as gray dust or evolution of the white dwarf progenitors is the culprit, we expect that the deviation of the apparent magnitude will continue growing, unless the systematic bias is set up in such an unlikely way as to mimic the effects of $\Lambda$ (Drell et al. 2000). A turnover, or decrease of the deviation of apparent magnitude at high redshift, can be considered almost like a "smoking gun" of $\Lambda$ (or, more generally, of a dark-energy component whose density does not change much with time). 
In a wonderful demonstration of good luck and hard work, Riess et al. (2001) report on $H S T$ observations of a probable SN Ia at $z \approx 1.7$ (SN $1997 \mathrm{ff}$, the most distant SN ever observed) that suggest the expected turnover is indeed present, providing a tantalizing glimpse of the epoch of deceleration. (See also Benítez et al. 2002, which corrects the observed magnitude of SN 1997ff for gravitational lensing by foreground galaxies.) SN 1997ff was discovered by Gilliland \& Phillips (1998) in a repeat HST observation of the Hubble Deep Field-North, and it was serendipitously monitored in the infrared with $H S T$ /NICMOS. The peak apparent SN brightness is consistent with that expected in the decelerating phase of the concordance cosmological model, $\Omega_{M} \approx 0.3, \Omega_{\Lambda} \approx 0.7$ (Figure 12). It is inconsistent with gray dust or simple luminosity evolution, when combined with the data for SNe Ia at $z \approx 0.5$.

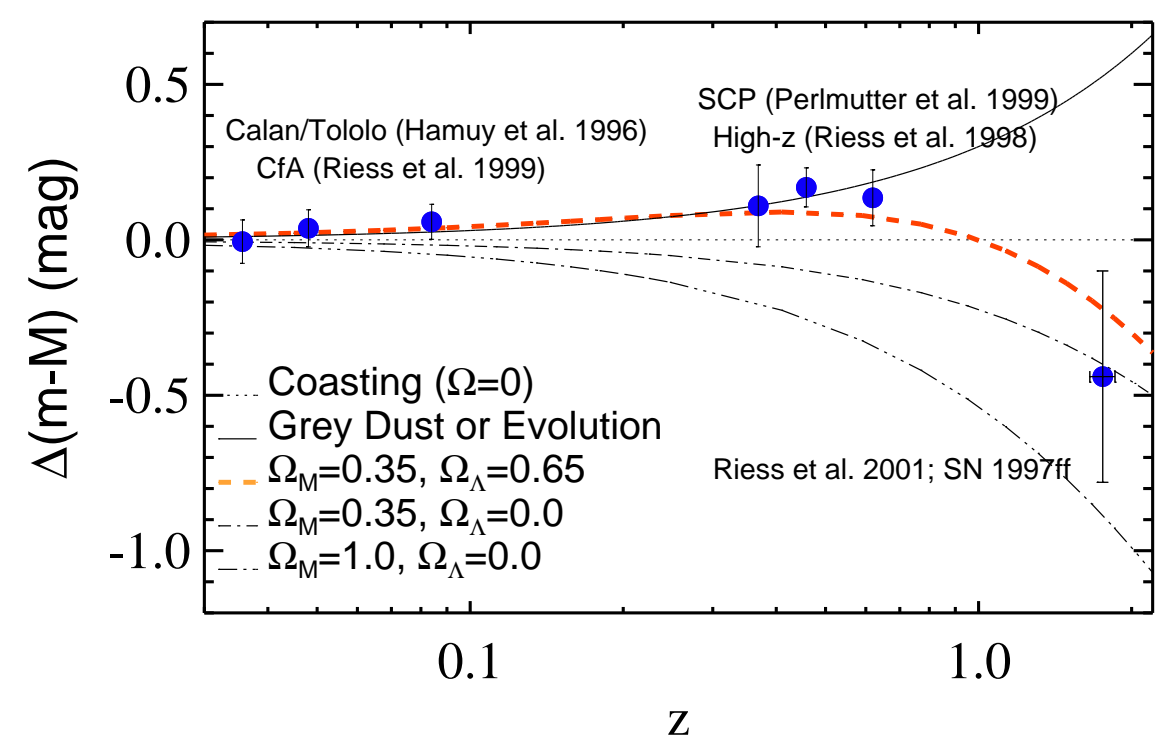

Figure 12: Hubble diagram for SNe Ia relative to an empty universe $(\Omega=0)$ compared with cosmological and astrophysical models (Riess et al. 2001). Low-redshift SNe Ia are from Hamuy et al. (1996a) and Riess et al. (1999a). The magnitude of SN 1997ff at $z=1.7$ has been corrected for gravitational lensing (Benítez et al. 2002). The measurements of SN 1997ff are inconsistent with astrophysical effects that could mimic previous evidence for an accelerating universe from SNe Ia at $z \approx 0.5$.

On the other hand, it was wise to remain cautious at the time: the error bars are large, and it is always possible that we are being fooled by this single object. The HZT and SCP thus started programs to find and measure more $\mathrm{SNe}$ Ia at such high redshifts $(z>1)$. One promising- 
looking result was that of Tonry et al. (2003) and Barris et al. (2004) for the HZT: the deviation of apparent magnitude from the low- $\Omega_{M}$, zero- $\Lambda$ model for several new SNe Ia at $z \approx 1$ is roughly the same as that at $z \approx 0.5$, in agreement with expectations based on the results of Riess et al. (2001).

Inspired by his promising results from SN 1997ff, as well as by the discovery and study of two distant SNe Ia (Blakeslee et al. 2003) with the HST Advanced Camera for Surveys (ACS), Adam Riess launched an extensive HST campaign (the "Hubble Higher- $z$ Supernova Search") to closely monitor $\sim 6$ high-redshift $(z \gtrsim 1)$ SNe Ia, with the goal of more clearly detecting the early epoch of deceleration. A major obstacle was the vast amount of HST time required to both discover and obtain followup photometry of the faint SNe, but this was overcome by arranging to "piggyback" on the Great Observatories Origins Deep Survey (GOODS), in which $\sim 400 H S T$ orbits were being used to obtain deep, multicolor ACS images of distant galaxies. The GOODS team obtained their data in several distinct epochs with separations of 45 days, and with suitable filters, allowing the discovery of high-redshift $\mathrm{SNe}$ (e.g., Giavalisco et al. 2002; Riess et al. 2004a), generally on the rise. Results from Jha (2002) suggested that key light-curve-shape parameters, such as the time of maximum and decline rate, could be determined from rest-frame $U$ band light curves, corresponding to observed-frame ACS $z$-band images, allowing for fewer epochs of costly infrared (NICMOS) observations to measure the SN luminosity and color. This strategy allowed the requisite follow-up images (e.g., Figure 13) and ACS grism spectra to be obtained with a relatively modest award of additional HST time.

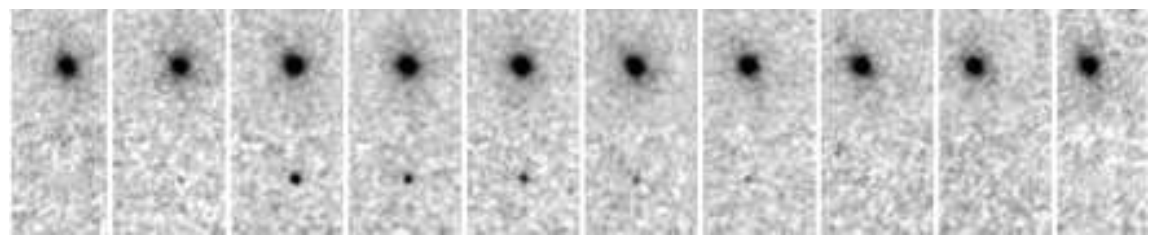

Figure 13: SN 2002hp, a high-redshift supernova from the GOODS program using HST. One can see it brightening and subsequently fading with time. The assumed host galaxy is at the top of each frame.

The program was very successful. Riess et al. (2004b; see also Strolger et al. 2004) convincingly show that SNe Ia at $z \gtrsim 1$ are brighter than predicted in simple models invoking systematic effects (Fig. 14). Thus, many SN Ia evolution and dust models are excluded as alternatives to acceleration for the observed faintness of $z \approx 0.5 \mathrm{SNe}$ Ia. The data demonstrate that the early universe was indeed apparently decelerating, 
as expected if $\Lambda$ is a relatively recent effect (Fig. 15). The expansion of the Universe made a transition between deceleration and acceleration (Turner \& Riess 2002 ) at $z \approx 0.5$, consistent with the new "concordance cosmology" of $\Omega_{M} \approx 0.27, \Omega_{\Lambda} \approx 0.73$ from the best CMB measurements (Spergel et al. 2003) and (independently) the complete set of available SN Ia data (Riess et al. 2004b). Finally, Riess et al. (2004b) show that the value of the dark energy equation-of-state parameter is consistent with $w=-1$, and that changes in $w$ could not have been very large over the past $\sim 10$ billion years (i.e., $d w / d z=w^{\prime} \approx 0$, as expected with $\Lambda$ ). Even better agreement with the $\Lambda$-model expectations was obtained by Wang \& Tegmark (2004), who analyzed the data in a slightly different manner. Thus, although we cannot exclude the possibility that the Universe will recollapse in the future, such a "big crunch" (or "gnaB giB," which is "Big Bang" backwards) is unlikely to occur in fewer than 15-30 Gyr (Riess et al. 2004b) if we adopt the linear potential field of Kallosh \& Linde (2003).

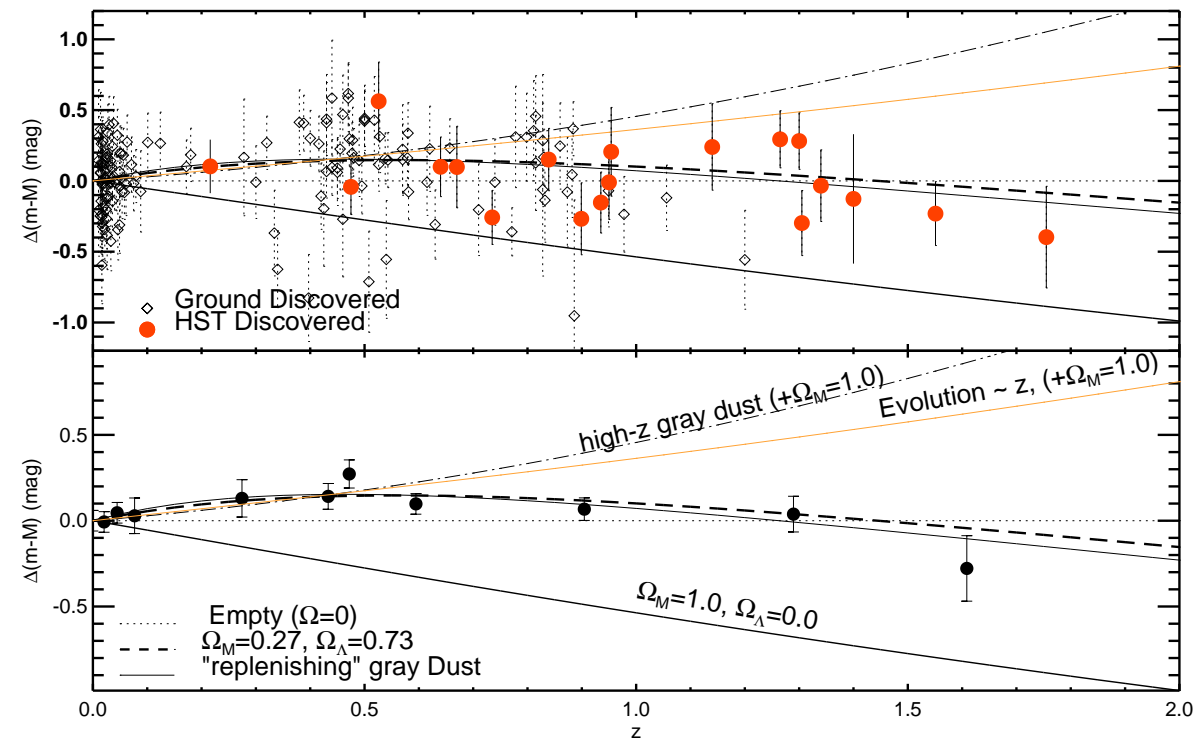

Figure 14: SN Ia residual Hubble diagram comparing cosmological models and scenarios for astrophysical dimming (Riess et al. 2004b). Upper panel: SNe Ia from ground-based discoveries are shown as diamonds, HST-discovered SNe Ia are shown as filled symbols. Lower panel: Weighted averages in fixed redshift bins, which are given only for illustrative purposes. Data and models are shown relative to an empty universe model $(\Omega=0)$. 


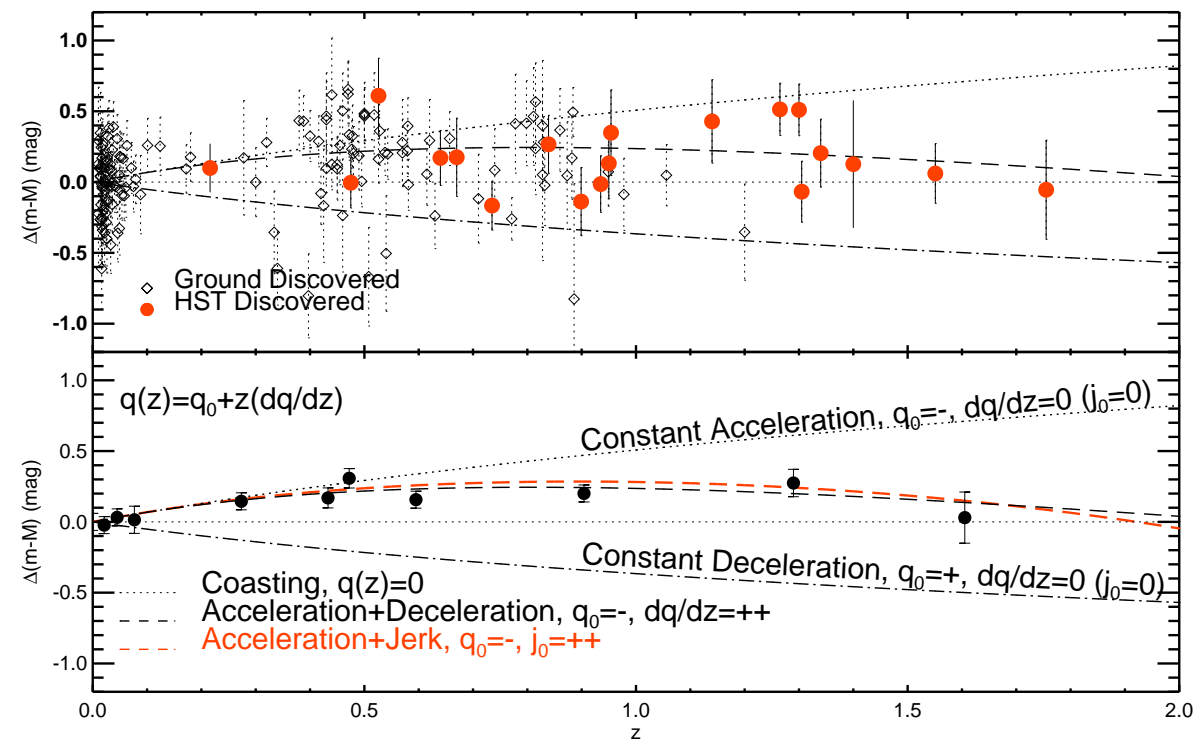

Figure 15: Kinematic SN Ia residual Hubble diagram (Riess et al. 2004b). Upper panel: Symbols as in Figure 14. Lower panel: Weighted averages, as in Fig. 14. Data and kinematic models of the expansion history are shown relative to an eternally coasting model, $q(z)=0$. Models representing specific kinematic scenarios (e.g., "constant acceleration") are illustrated.

Of course, it is possible to find specific dust or evolution models that are not ruled out with our SN Ia data. For example, a "replenishing dust" model represents a constant density of dust that is continually replenished at precisely the rate at which it is diluted by the expanding universe (Goobar, Bergstrom, \& Mortsell 2002; Riess et al. 2004b); one could perhaps call this the "steady-state dust model." Since the dimming is directly proportional to the distance light traveled and is thus mathematically quite similar to the effects of a cosmological constant, we cannot discriminate this model from the $\Lambda$-dominated model in the magnitude-redshift plane. However, the fine-tuning required of this dust's opacity, replenishing rate, and velocity ( $\gtrsim 1000 \mathrm{~km} \mathrm{~s}^{-1}$ for it to fill space uniformly without adding detectable dispersion) makes it unattractive as a simpler alternative to a cosmological constant. Moreover, the density of intergalactic dust needed to explain the observed faintness of SNe Ia at $z \approx 0.5$ exceeds the upper limit determined by 
Paerels et al. (2002) from the absence of a detectable X-ray scattering halo around a single distant quasar.

Another model with this behavior is evolution that is proportional to look-back time (Wright 2001). While possible, such dimming behavior, especially if in the form of luminosity evolution, seems implausible. We may expect evolution (or dust production) to be coupled to the observed evolution of stellar populations, galaxies morphologies, sizes, large-scale structure, or even chemical enrichment. None of these known varieties of evolution are largely completed by $z=0.5$ starting from their properties at $z=0$; quite the contrary, most of them have hardly begun, looking back to $z=0.5$. As mentioned previously, a strong empirical argument against recent luminosity evolution is the independence of SN Ia distance measurements on gross host morphology (Riess et al. 1998b; Sullivan et al. 2003). The range of progenitor formation environments spanned by SNe Ia in early-type and late-type hosts greatly exceeds any evolution in the mean host properties between $z=0$ and $z=0.5$. In the end, however, the only "proof" against astrophysical contamination of the cosmological signal from $\mathrm{SNe}$ Ia is to test the results against those of other observations, independent of SNe Ia.

\subsection{Type Ia Supernova Rates and Progenitors}

The rate of SNe Ia as a function of redshift is important to know for a number of reasons. For example, it affects the derived chemical evolution of galaxies (e.g., Maoz \& Gal-Yam 2004; Matteucci \& Recchi 2001, and references therein), and it can be used to set constraints on the star formation history of the Universe (Gal-Yam \& Maoz 2004). Moreover, the distribution of "delay times" between the formation of the progenitor star and the explosion of the supernova can be determined from an accurate census of the SN Ia rate at different cosmic times, thereby providing clues to the physical nature of the progenitors of SNe Ia (Madau, Della Valle, \& Panagia 1998; Dahlen \& Fransson 1999; Yungelson \& Livio 2000). There have been many measurements of the rate of very nearby SNe Ia (Cappellaro, Evans, \& Turatto 1999, and references therein), and at $z \approx 0.1$ several studies have been conducted (Hardin et al. 2000; Strolger 2003; Reiss 2000). These form the basis of comparison for the measurements at higher redshifts.

From observations of three SNe Ia at $z \approx 0.4$, Pain et al. (1996) estimated a rate of SNe Ia equivalent to $34 \mathrm{yr}^{-1} \mathrm{deg}^{-2}$, with a $1 \sigma$ uncertainty of a factor of $\sim 2$, for objects found in the range of $21.3<$ $R<22.3$ mag. A more recent estimate by Pain et al. (2002a) is $(1.53 \pm 0.3) \times 10^{-4} h^{3} \mathrm{Mpc}^{-3} \mathrm{yr}^{-1}$ at a mean redshift of 0.55 (where 
$h \equiv H_{0} /\left(100 \mathrm{~km} \mathrm{~s}^{-1} \mathrm{Mpc}^{-1}\right)$. Cappellaro et al. (1999) report a nearby $\mathrm{SN}$ Ia rate of $0.36 \pm 0.11 h^{2} \mathrm{SNu}$ (where $1 \mathrm{SNu} \equiv 10^{10} L_{B_{\odot}}$ per century), or $(0.79 \pm 0.24) \times 10^{-4} h^{3} \mathrm{Mpc}^{-3} \mathrm{yr}^{-1}$, using a quite uncertain local luminosity density of $\rho=2.2 \times 10^{8} h L_{B} \mathrm{Mpc}^{-3}$. Pain et al. (2002a) claim to see a very modest increase in the rate of SNe Ia with redshift, perhaps tracking the star formation rate which Wilson et al. (2002) estimate as being proportional to $(1+z)^{1.7}$ in a flat universe with $\Omega_{M}=0.3$.

Based on a new sample of SNe Ia observed during the 1999 HZT campaign, an independent estimate of the SN Ia rate at a mean redshift of 0.46 was made by Tonry et al. (2003): $(1.4 \pm 0.5) \times 10^{-4} h^{3} \mathrm{Mpc}^{-3}$ $\mathrm{yr}^{-1}$. This is in excellent agreement with the results of Pain et al. (2002a), and it is not inconsistent with the local rates from Cappellaro et al. (1999), particularly given the uncertainty in the local luminosity density. On the other hand, from a different sample of SNe Ia (see below), Dahlen et al. (2004) find a somewhat higher rate of SNe Ia at $z \approx 0.4$ and suggest that the previously published rates may have been underestimated due to systematic effects. Indeed, B. Barris (2004, private communication) has found evidence that the true rate of SNe Ia in the 2001 HZT campaign is higher than that reported by Barris et al. (2004); similar biases may have affected the 1999 HZT campaign and other previous searches.

Although Tonry et al. (2003) were incomplete in their counts of SNe Ia at $z \approx 1$, they do not believe that the rate of SNe Ia closely tracks the star formation rate. Application of the star formation rate from Wilson et al. (2002) would suggest that the SN Ia rate at $z \approx 1.1$ should be three times as great as occurs locally and nearly twice as great as the rate at $z=0.46$. In this case, Tonry et al. (2003) should have discovered $16 \mathrm{SNe} \mathrm{Ia} \mathrm{deg}^{-2}$ in their search, yet they only found $4 \mathrm{SNe} \mathrm{Ia} \mathrm{deg}^{-2}$. It is their impression that the constant rate per volume is closer to the truth.

The more recent results of Dahlen et al. (2004) conflict with this conclusion. They determine the rate of SNe Ia as a function of redshift by using data from the Hubble Higher- $z$ Supernova Search of Riess et al. (2004b), with a redshift range of $0.2<z<1.6$. The resulting SN Ia rate at $z \approx 1$ is a factor of $3-5$ higher than previous estimates made at lower redshifts $(z<0.5)$, presumably because the star formation rate at $z>1$ was substantially higher than that in the past few Gyr. Moreover, their data suggest that at even higher redshifts, $z>1$, the rate of SNe Ia begins to decrease. They find that the delay time (from progenitor star formation to SN Ia explosion) is likely to be substantial, $\gtrsim 2$ Gyr. In addition, assuming a Salpeter (1955) initial mass function and a SN Ia progenitor main-sequence mass range of 3-8 $M_{\odot}$, it appears that $5-7 \%$ of 
the white dwarfs formed from these progenitor stars eventually become SNe Ia (but fewer than $1 \%$ of all white dwarfs eventually become SNe Ia).

Strolger et al. (2004) use the above results, together with additional modeling, to set more quantitative constraints on the nature of the progenitors of SNe Ia. Specifically, they use a Bayesian maximum likelihood test to determine the most likely range of delay times that best reproduces the observed redshift distribution of SNe Ia. They find that models requiring a large fraction of "prompt" (less than 2 Gyr) SNe Ia poorly reproduce the observed redshift distribution and are rejected at $>99 \%$ confidence. Thus, SNe Ia cannot generally be prompt events, nor can they be expected to closely follow the star formation rate history. Instead, Gaussian models best fit the observed data for mean delay times in the range of 3-4 Gyr. This may be most consistent with single-degenerate systems in which the white dwarf accretes from a main-sequence companion or from a somewhat evolved companion (Livio 2001), although certain types of double-degenerate models are not yet eliminated. Tests conducted by Gal-Yam \& Maoz (2004) also conclude that the characteristic delay times of SNe Ia should be large ( $>1-2$ Gyr) for similar assumed models of the star formation rate history, but the results are not as definitive as those of Strolger et al. (2004) because they are based on SN Ia rates derived from more limited SCP data (Pain et al. 2002a).

\subsection{Measuring the Dark Energy Equation-of-State Parameter}

Every energy component in the Universe can be parameterized by the way its density varies as the Universe expands (scale factor $a$ ), with $\rho \propto a^{-3(1+w)}$, and $w$ is the component's equation-of-state parameter, $w=P /\left(\rho c^{2}\right)$, where $P$ is the pressure exerted by the component. So for matter, $w=0$, while an energy component that does not vary with scale factor has $w=-1$, as in the cosmological constant $\Lambda$. Quintessence models have $w \neq-1$, and generally $d w / d z \neq 0)$. Some really strange energies may have $w<-1$ : their density increases with time (Carroll, Hoffman, \& Trodden 2003), leading to a "Big Rip" in which progressively smaller bound systems get torn apart! [Riess et al. (2004b) and Caldwell et al. (2003) estimate that such a fate will not occur sooner than $\sim 20$ Gyr from now, if ever.] Clearly, a good estimate of $w$ becomes the key to differentiating between models.

The CMB observations imply that the geometry of the universe is close to flat, so the energy density of the dark component is simply related to the matter density by $\Omega_{x}=1-\Omega_{M}$. This allows the luminosity distance 
as a function of redshift to be written as

$$
D_{L}(z)=\frac{c(1+z)}{H_{0}} \int_{0}^{z} \frac{\left[1+\Omega_{x}\left((1+\mathrm{z})^{3 w}-1\right)\right]^{-1 / 2}}{(1+\mathrm{z})^{3 / 2}} \mathrm{dz},
$$

showing that the dark energy density and equation of state directly influence the apparent brightness of standard candles. As demonstrated graphically in Figure 16, SNe Ia observed over a wide range of redshifts can constrain the dark energy parameters to a cosmologically interesting accuracy.

But there are two major problems with using SNe Ia to measure $w$. First, systematic uncertainties in SN Ia peak luminosity limit how well $D_{L}(z)$ can be measured. While statistical uncertainty can be arbitrarily reduced by finding thousands of SNe Ia, intrinsic SN properties such as evolution and progenitor metallicity, and observational limits like photometric calibrations and K-corrections, create a systematic floor that cannot be decreased by sheer force of numbers. We expect that systematics can be controlled to at best $3 \%$, with considerable effort.

Second, SNe at $z>1.0$ are very hard to discover and study from the ground. As discussed above, both the HZT and the SCP have found a few SNe Ia at $z>1.0$, but the numbers and quality of these light curves are insufficient for a $w$ measurement. Large numbers of SNe Ia at $z>1.0$ are best left to a wide-field optical/infrared imager in space, such as the proposed Supernova/ Acceleration Probe (SNAP; Nugent et al. 2001) satellite.

Fortunately, an interesting measurement of $w$ can be made at present. The current values of $\Omega_{M}$ from many methods (most recently WMAP: 0.27; Spergel et al. 2003) make an excellent substitute for those expensive $\mathrm{SNe}$ at $z>1.0$. Figure 16 shows that a $\mathrm{SN}$ Ia sample with a maximum redshift of $z=0.8$, combined with the current $10 \%$ error on $\Omega_{M}$, will do as well as a SN Ia sample at much higher redshifts. Within a few years, the Sloan Digital Sky Survey and WMAP will solidify the estimate of $\Omega_{M}$ and sharpen $w$ further.

Both the SCP and the HZT are involved in multi-year programs to discover and monitor hundreds of SNe Ia for the purpose of measuring $w$. For example, the HZT's project, ESSENCE (Equation of State: SupErNovae trace Cosmic Expansion), is designed to discover $200 \mathrm{SNe}$ Ia evenly distributed in the $0.2<z<0.8$ range (Smith et al. 2002; Garnavich et al. 2002; http://www.ctio.noao.edu/wproject). The CTIO 4-m telescope and mosaic camera are being used to find and follow the SNe by imaging on every other dark night for several consecutive months of the year. Keck and other large telescopes are being used to get the SN spectra and redshifts. Project ESSENCE will eventually provide an 
estimate of $w$ to an accuracy of $\sim 10 \%$ (Figure 17). Even larger numbers of high-redshift SNe Ia are being found during the ongoing CFHT Legacy Survey (http://www.cfht.hawaii.edu/Science/CFHLS; Pain et al. 2002b), providing an independent sample with which to measure the value of $w$. Within the next few years, Pan-STARRS (Kaiser et al. 2002; http://poi.ifa.hawaii.edu) should discover and follow thousands of SNe Ia.
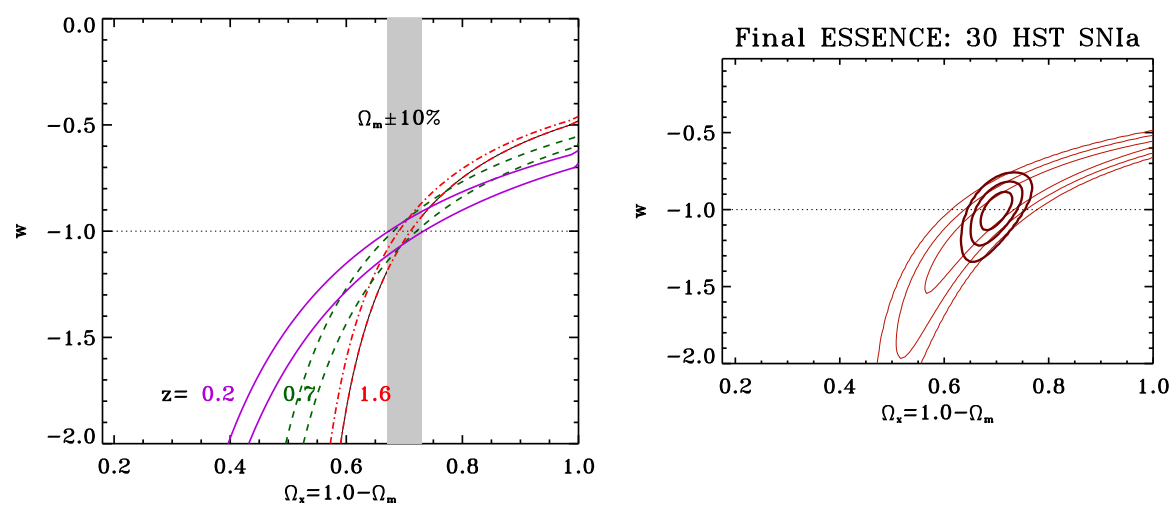

Figure 16 (left): Constraints on $\Omega_{x}$ and $w$ from SN data sets collected at $z=0.2$ (solid lines), $z=0.7$ (dashed lines), and $z=1.6$ (dash-dot lines). The shaded area indicates how an independent estimate of $\Omega_{M}$ with a $10 \%$ error can help constrain $w$.

Figure 17 (right): Expected constraints on $w$ with the desired final ESSENCE data set of $200 \mathrm{SNe}$ Ia, 30 of which (in the redshift range $0.6<z<0.8)$ are to be observed with $H S T$. The thin lines are for SNe alone while the thick lines assume an uncertainty in $\Omega_{M}$ of $7 \%$. The final ESSENCE data will constrain the value of $w$ to $\sim 10 \%$.

Farther in the future, the plethora of SNe Ia to be found and studied by the proposed SNAP satellite (Nugent et al. 2001), the NASA/DOE Joint Dark Energy Mission (JDEM), the Large-area Synoptic Survey Telescope (the "Dark Matter Telescope"; Tyson \& Angel 2001), and similar large-scale projects could reveal whether the value of $w$ depends on redshift, and hence should give additional constraints on the nature of the dark energy. High-redshift surveys of galaxies such as DEEP2 (Davis et al. 2001), as well as space-based missions to map the CMB (Planck), should provide additional evidence for (or against) $\Lambda$. Observational cosmology promises to remain exciting for quite some time! 


\section{Acknowledgments}

I thank all of my HZT collaborators for their contributions to our team's research, and members of the SCP for their seminal complementary work on the accelerating Universe. My group's work at U.C. Berkeley has been supported by NSF grants AST-9987438, AST-0206329, and AST-0307894, as well as by grants GO-7505, GO/DD-7588, GO-8177, GO-8641, GO-9118, and GO-9352 from the Space Telescope Science Institute, which is operated by the Association of Universities for Research in Astronomy, Inc., under NASA contract NAS 5-26555. Many spectra of high-redshift SNe were obtained at the W. M. Keck Observatory, which is operated as a scientific partnership among the California Institute of Technology, the University of California, and NASA; the observatory was made possible by the generous financial support of the W. M. Keck Foundation. KAIT has received donations from Sun Microsystems, Inc., the Hewlett-Packard Company, AutoScope Corporation, Lick Observatory, the National Science Foundation, the University of California, and the Sylvia and Jim Katzman Foundation. I am grateful to the editors of this volume for their incredible patience while waiting for my review.

\section{References}

Afshordi, N., Loh, Y.-S., \& Strauss, M. S. 2004, Phys. Rev. D, 69, 083524

Aguirre, A. N. 1999a, ApJ, 512, L19

Aguirre, A. N. 1999b, ApJ, 525, 583

Aguirre, A. N., \& Haiman, Z. 1999, ApJ, 525, 583

Aldering, G., Knop, R., \& Nugent, P. 2000, AJ, 119, 2110

Bahcall, J. N., et al. 1996, ApJ, 457, 19

Bahcall, N. A., Ostriker, J. P., Perlmutter, S., \& Steinhardt, P. J. 1999, Science, 284, 1481

Balbi, A., et al. 2000, ApJ, 545, L1

Barber, A. J., Thomas, P. A., Couchman, H. M. P., \& Fluke, C. J. 2000, MNRAS, 319, 267

Barris, B., et al. 2004, ApJ, 602, 571

Benítez, N., Riess, A., Nugent, P., Dickinson, M., Chornock, R., \& Filippenko, A. V. 2002, ApJ, 577, L1

Blakeslee, J. P., et al. 2003, ApJ, 589, 693

Boughn, S., \& Crittenden, R. 2004, Nature, 427, 45

Branch, D. 1981, ApJ, 248, 1076

Branch, D. 1998, ARAEAA, 36, 17

Branch, D., Baron, E., Thomas, R. C., Kasen, D., Li, W., \& Filippenko, A. V. 2004b, $P A S P$, in press (astro-ph/0408130)

Branch, D., Fisher, A., \& Nugent, P. 1993, AJ, 106, 2383

Branch, D., \& Miller, D. L. 1993, ApJ, 405, L5

Branch, D., Romanishin, W., \& Baron, E. 1996, ApJ, 465, 73 (erratum: 467, 473)

Branch, D., \& Tammann, G. A. 1992, ARA\&A, 30, 359 
Branch, D., et al. 2004a, ApJ, 606, 413

Caldwell, R. R., Davé, R., \& Steinhardt, P. J. 1998, Ap\&SSS, 261, 30

Caldwell, R. R., Kamionkowski, M., \& Weinberg, N. N. 2003, Phys. Rev. Lett., 91, 71301

Cappellaro, E., Evans, R., \& Turatto, M. 1999, A\&\&A, 351, 459

Cappellaro, E., Turatto, M., Tsvetkov, D. Yu., Bartunov, O. S., Pollas, C., Evans, R., \& Hamuy, M. 1997, A\& A, 322, 431

Carroll, S. M., Hoffman, M., \& Trodden, M. 2003, Phys. Rev. D, 68, 023509

Carroll, S. M., Press, W. H., \& Turner, E. L. 1992, ARA\& A, 30, 499

Chaboyer, B., Demarque, P., Kernan, P. J., \& Krauss, L. M. 1998, ApJ, 494, 96

Coil, A. L., et al. 2000, ApJ, 544, L111

Cowan, J. J., McWilliam, A., Sneden, C., \& Burris, D. L. 1997, ApJ, 480, 246

Dahlen, T., \& Fransson, C. 1999, A\& A, 350, 349

Dahlen, T., et al. 2004, ApJ, 613, 189

Davis, M., Newman, J. A., Faber, S. M., \& Phillips, A. C. 2001, in Deep Fields, ed.

S. Cristiani, A. Renzini, \& R. E. Williams (Berlin: Springer), 241

de Bernardis, P., et al. 2000, Nature, 404, 955

de Bernardis, P., et al. 2002, ApJ, 564, 559

Drell, P. S., Loredo, T. J., \& Wasserman, I. 2000, ApJ, 530, 593

Efstathiou, G., et al. 1999, MNRAS, 303, L47

Efstathiou, G., et al. 2002, MNRAS, 330, L29

Eisenstein, D. J., Hu, W., \& Tegmark, M. 1998, ApJ, 504, L57

Falco, E. E., et al. 1999, ApJ, 523, 617

Filippenko, A. V. 1997a, in Thermonuclear Supernovae, ed. P. Ruiz-Lapuente et al. (Dordrecht: Kluwer), 1

Filippenko, A. V. 1997b, ARA\&A, 35, 309

Filippenko, A. V. 2001, PASP, 113, 1441

Filippenko, A. V. 2003, in From Twilight to Highlight: The Physics of Supernovae, ed. W. Hillebrandt \& B. Leibundgut (Berlin: Springer-Verlag), 171

Filippenko, A. V., Li, W. D., Treffers, R. R., \& Modjaz, M. 2001, in Small-Telescope Astronomy on Global Scales, ed. W. P. Chen, C. Lemme, \& B. Paczyński (San Francisco: ASP), 121

Filippenko, A. V., \& Riess, A. G. 1998, Phys. Rep., 307, 31

Filippenko, A. V., et al. 1992a, $A J, \mathbf{1 0 4}, 1543$

Filippenko, A. V. 1992b, ApJ, 384, L15

Foley, R. J., et al. 2003, PASP, 115, 1220

Ford, C. H., et al. 1993, $A J, \mathbf{1 0 6}, 1101$

Fosalba, P., et al. 2003, $A p J, \mathbf{5 9 7}$, L89

Freedman, W., et al. 2001, ApJ, 553, 47

Gal-Yam, A., \& Maoz, D. 2004, MNRAS, 347, 942

Garnavich, P., et al. 1998a, ApJ, 493, L53

Garnavich, P., et al. 1998b, ApJ, 509, 74

Garnavich, P., et al. 2002, BAAS, 34, 1233

Giavalisco, M., et al. 2002, IAUC 7981

Gibson, B. K., et al. 2000, ApJ, 529, 723

Gilliland, R. L., \& Phillips, M. M. 1998, IAUC 6810

Goldhaber, G., \& Perlmutter, S. 1998, Phys. Rep., 307, 325

Goldhaber, G., et al. 1997, in Thermonuclear Supernovae, ed. P. Ruiz-Lapuente et al.

(Dordrecht: Kluwer), 777

Goldhaber, G., et al. 1998a, BAAS, 30, 1325 
Goldhaber, G., et al. 1998b, in Gravity: From the Hubble Length to the Planck Length, SLAC Summer Institute (Stanford, CA: SLAC)

Goldhaber, G., et al. 2001, $A p J, \mathbf{5 5 8}, 359$

Goobar, A., Bergstrom, L., \& Mortsell, E. 2002, A\&A, 384, 1

Goobar, A., \& Perlmutter, S. 1995, ApJ, 450, 14

Gratton, R. G., Fusi Pecci, F., Carretta, E., Clementini, G., Corsi, C. E., \& Lattanzi, M. 1997, ApJ, 491, 749

Groom, D. E. 1998, BAAS, 30, 1419

Hamuy, M., Phillips, M. M., Maza, J., Suntzeff, N. B., Schommer, R. A., \& Aviles, R. 1995, AJ, 109, 1

Hamuy, M., Phillips, M. M., Maza, J., Suntzeff, N. B., Schommer, R. A., \& Aviles, R. 1996a, AJ, 112, 2391

Hamuy, M., Phillips, M. M., Maza, J., Suntzeff, N. B., Schommer, R. A., \& Aviles, R. 1996b, AJ, 112, 2398

Hamuy, M., Phillips, M. M., Maza, J., Suntzeff, N. B., Schommer, R. A., \& Aviles, R. 1996c, AJ, 112, 2408

Hamuy, M., Trager, S. C., Pinto, P. A., Phillips, M. M., Schommer, R. A., Ivanov, V., \& Suntzeff, N. B. 2000, $A J, \mathbf{1 2 0}, 1479$

Hanany, S., et al. 2000, $A p J, \mathbf{5 4 5}$, L5

Hancock, S., Rocha, G., Lazenby, A. N., \& Gutiérrez, C. M. 1998, MNRAS, 294, L1

Hardin, D., et al. 2000, $A \mathscr{E} A, \mathbf{3 6 2}, 419$

Hatano, K., Branch, D., \& Deaton, J. 1998, ApJ, 502, 177

Höflich, P., Wheeler, J. C., \& Thielemann, F. K. 1998, ApJ, 495, 617

Holz, D. E. 1998, ApJ, 506, L1

Holz, D. E., \& Wald, R. 1998, Phys. Rev. D, 58, 063501

Hoyle, F., Burbidge, G., \& Narlikar, J. V. 2000, A Different Approach to Cosmology (Cambridge: Cambridge Univ. Press)

Ivanov, V. D., Hamuy, M., \& Pinto, P. A. 2000, ApJ, 542, 588

Jha, S. 2002, Ph.D. thesis, Harvard University

Kaiser, N., et al. 2002, BAAS, 34, 1304

Kallosh, R., \& Linde, A. 2003, J. Cosmology Astropart. Phys., 2, 2

Kantowski, R. 1998, ApJ, 507, 483

Kantowski, R., Vaughan, T., \& Branch, D. 1995, ApJ, 447, 35

Kim, A., Goobar, A., \& Perlmutter, S. 1996, PASP, 108, 190

Knop, R., et al. 2003, ApJ, 598, 102

Krisciunas, K., Phillips, M. M., \& Suntzeff, N. 2004, ApJ, 602, L81

Krisciunas, K., et al. 2001, $A J, \mathbf{1 2 2}, 1616$

Krisciunas, K., et al. 2003, $A J, \mathbf{1 2 5}, 166$

Krisciunas, K., et al. 2004, $A J, \mathbf{1 2 7}, 1664$

Leibundgut, B. 2001, ARA $\dot{E} A, \mathbf{3 9}, 67$

Leibundgut, B., et al. 1993, $A J, \mathbf{1 0 5}, 301$

Leibundgut, B., et al. 1996, $A p J$, 466, L21

Leonard, D. C., et al. 2002a, PASP, 114, 35 (erratum: 114, 1291)

Leonard, D. C., et al. 2002b, AJ, 124, 2490

Li, W., Filippenko, A. V., Chornock, R., \& Jha, S. 2003a, ApJ, 586, L9

Li, W., Filippenko, A. V., Chornock, R., \& Jha, S. 2003c, PASP, 115, 844

Li, W., Filippenko, A. V., Treffers, R. R., Riess, A. G., Hu, J., \& Qiu, Y. 2001b, ApJ, 546, 734

Li, W., et al. 2000, in Cosmic Explosions, ed. S. S. Holt \& W. W. Zhang (New York: AIP), 103 
Li, W., et al. 2001a, PASP, 113, 1178

Li, W., et al. 2003b, $P A S P, \mathbf{1 1 5}, 453$

Lineweaver, C. H. 1998, ApJ, 505, L69

Lineweaver, C. H., \& Barbosa, D. 1998, ApJ, 496, 624

Livio, M. 2001, in Supernovae and Gamma-Ray Bursts: The Greatest Explosions since the Big Bang, ed. K. Sahu, M. Livio, \& N. Panagia (Cambridge: Cambridge Univ. Press), 334

Madau, P., Della Valle, M., \& Panagia, N. 1998, MNRAS, 297, L17

Maoz, D., \& Gal-Yam, A. 2004, MNRAS, 347, 951

Matheson, T., Filippenko, A. V., Li, W., Leonard, D. C., \& Shields, J. C. 2001, AJ, 121, 1648

Matheson, T., et al. 2003, ApJ, 599, 394

Matteucci, F., \& Recchi, S. 2001, ApJ, 558, 351

Modjaz, M., Li, W., Filippenko, A. V., King, J. Y., Leonard, D. C., Matheson, T., Treffers, R. R., \& Riess, A. G. 2001, PASP, 113, 308

Narlikar, J. V., \& Arp, H. C. 1997, ApJ, 482, L119

Netterfield, C. B., et al. 2002, ApJ, 571, 604

Nolta, M. R., et al. 2004, ApJ, 608, 10

Nomoto, K., Umeda, H., Hachisu, I., Kato, M., Kobayashi, C., \& Tsujimoto, T. 2000, in Type Ia Supernovae: Theory and Cosmology, ed. J. C. Niemeyer \& J. W. Truran (Cambridge: Cambridge Univ. Press), 63

Norgaard-Nielsen, H., et al. 1989, Nature, 339, 523

Nugent, P., 2001, in Particle Physics and Cosmology: Second Tropical Workshop, ed. J. F. Nieves (New York: AIP), 263

Nugent, P., Kim, A., \& Perlmutter, S. 2002, PASP, 114, 803

Nugent, P., Phillips, M., Baron, E., Branch, D., \& Hauschildt, P. 1995, ApJ, 455, L147

Ostriker, J. P., \& Steinhardt, P. J. 1995, Nature, 377, 600

Oswalt, T. D., Smith, J. A., Wood, M. A., \& Hintzen, P. 1996, Nature, 382, 692

Paerels, F., Petric, A., Telis, G., \& Helfand, D. J. 2002, BAAS, 34, 1264

Pain, R., et al. 1996, ApJ, 473, 356

Pain, R., et al. 2002a, $A p J, \mathbf{5 7 7}, 120$

Pain, R., et al. 2002b, $B A A S, \mathbf{3 4}, 1169$

Parodi, B. R., et al. 2000, ApJ, 540, 634

Peacock, J. A., et al. 2001, Nature, 410, 169

Percival, W., et al. 2001, MNRAS, 327, 1297

Perlmutter, S., et al. 1995a, ApJ, 440, L41

Perlmutter, S., et al. 1995b, IAUC 6270

Perlmutter, S., et al. 1997, ApJ, 483, 565

Perlmutter, S., et al. 1998, Nature, 391, 51

Perlmutter, S., et al. 1999, ApJ, 517, 565

Phillips, M. M. 1993, ApJ, 413, L105

Phillips, M. M., et al. 1992, AJ, 103, 1632

Phillips, M. M., et al. 1999, AJ, 118, 1766

Pskovskii, Yu. P. 1977, Sov. Astron., 21, 675

Pskovskii, Yu. P. 1984, Sov. Astron., 28, 658

Reiss, D. 2000, PhD thesis, University of Washington

Riess, A. G., Filippenko, A. V., Li, W. D., \& Schmidt, B. P. 1999b, AJ, 118, 2668

Riess, A. G., Nugent, P. E., Filippenko, A. V., Kirshner, R. P., \& Perlmutter, S. 1998a, ApJ, 504, 935 
Riess, A. G., Press, W. H., \& Kirshner, R. P. 1995, ApJ, 438, L17

Riess, A. G., Press, W. H., \& Kirshner, R. P. 1996a, ApJ, 473, 88

Riess, A. G., Press, W. H., \& Kirshner, R. P. 1996b, ApJ, 473, 588

Riess, A. G., et al. 1997, AJ, 114, 722

Riess, A. G., et al. 1998b, AJ, 116, 1009

Riess, A. G., et al. 1999a, AJ, 117, 707

Riess, A. G., et al. 1999c, $A J, \mathbf{1 1 8}, 2675$

Riess, A. G., et al. 2000, ApJ, 536, 62

Riess, A. G., et al. 2001, ApJ, 560, 49

Riess, A. G., et al. 2004a, $A p J$, 600, L163

Riess, A. G., et al. 2004b, $A p J$, 607, 665

Ruiz-Lapuente, P., et al. 1992, ApJ, 387, L33

Saha, A., et al. 1997, ApJ, 486, 1

Saha, A., et al. 2001, $A p J, \mathbf{5 6 2}, 314$

Salpeter, E. E. 1955, ApJ, 121, 161

Sandage, A., \& Tammann, G. A. 1993, ApJ, 415, 1

Sandage, A., et al. 1996, ApJ, 460, L15

Schmidt, B. P., et al. 1998, ApJ, 507, 46

Scranton, R., et al. 2004, Phys. Rev. Lett., submitted (astro-ph/0307335)

Smith, R. C., et al. 2002, BAAS, 34, 1232

Spergel, D. N., et al. 2003, ApJS, 148, 175

Strolger, L. 2003, PhD thesis, University of Michigan

Strolger, L., et al. 2004, ApJ, 613, 200

Sullivan, M., et al. 2003, MNRAS, 340, 1057

Suntzeff, N. 1996, in Supernovae and Supernova Remnants, ed. R. McCray \& Z. Wang

(Cambridge: Cambridge Univ. Press), 41

Suntzeff, N., et al. 1996, IAUC 6490

Tonry, J. L., et al. 2003, ApJ, 594, 1

Tripp, R. 1997, $A \mathscr{E} A$, 325, 871

Tripp, R. 1998, A\&A, 331, 815

Turatto, M., et al. 1996, MNRAS, 283, 1

Turner, M. S., \& Riess, A. G. 2002, ApJ, 569, 18

Tyson, J. A., \& Angel, R. 2001, in The New Era of Wide Field Astronomy, ed. R. Clowes, et al. (San Francisco: ASP), 347

Umeda, H., et al. 1999, ApJ, 522, L43

van den Bergh, S., \& Pazder, J. 1992, ApJ, 390, 34

Vaughan, T. E., Branch, D., Miller, D. L., \& Perlmutter, S. 1995, ApJ, 439, 558

Wambsganss, J., Cen, R., \& Ostriker, J. P. 1998, ApJ, 494, 29

Wang, Y., \& Tegmark, M. 2004, Phys. Rev. Lett., 92, 241302

Williams, B., et al. 2003, AJ, 126, 2608

Wilson, G., Cowie, L. L., Barger, A. J., \& Burke, D. J. 2002, AJ, 124, 1258

Wright, E. L. 2001, BAAS, 34, 574

Yungelson, L. R., \& Livio, M. 2000, ApJ, 528, 108

Zaldarriaga, M., Spergel, D. N., \& Seljak, U. 1997, ApJ, 488, 1 